\title{
Wave-Wave Interactions in a Periodic Chain with Quadratic Nonlinearity
}

\author{
Smruti R. Panigrahi*a, Brian F. Feeny ${ }^{a}$, and Alejandro R. Diaz ${ }^{a}$ \\ ${ }^{a} 428$ S. Shaw Lane, Michigan State University, East Lansing, MI, 48824, USA
}

\begin{abstract}
Two waves are studied using perturbation analysis for their interactions in an one-dimensional periodic structure with quadratic nonlinearity. A first-order multiple-scales analysis along with numerical simulations on the full chain are used to understand the interaction of two waves when one is the sub- or super-harmonic of the other. The strength of quadratic nonlinearity affects the rate at which the energy is exchanged between the two waves. Depending on parameters and energy states, the interactions between the waves are periodic or whirling and result in quasi-periodic combined propagating waves with either phase drifts or weakly phase-locking properties. The analysis suggests the possibility of the existence of emergent wave harmonics. Due to quadratic nonlinearity, a very small amplitude subharmonic or superharmonic wave mode can drift in its phase, and then burst out with a larger amplitude as it circumnavigates a separatrix. Depending on the parameters and wave numbers, the amplitude of this emergent wave burst can have varying significance.
\end{abstract}

Keywords: Traveling waves, periodic structures, quadratic nonlinearity, perturbation, method of multiple scales, dispersion, metamaterials, acoustic filters, subharmonic, superharmonic, emergent waves, phase drift, phase lock.

\section{INTRODUCTION}

Due to the presence of bandgaps in periodic media, many researchers have been focusing on wave propagation in nonlinear periodic structures and its application to the design of novel metamaterials [1]-[8]. Structures exhibiting bandgaps prevent the propagation of waves at certain frequencies. These structures may be phononic (sonic) or photonic, depending on their band-gap frequency range. Phononic or sonic band-gap structures can be used as sensing devices based on resonators, acoustic logic ports and wave guides, and frequency filters based on surface acoustic waves, while photonic band-gap structures have applications in optics and microwaves. Synthesis of phononic materials with desired band-gap and wave-guiding characteristics has been achieved through the application of topology and material optimization procedures [9]-[11]. The application of periodic plane grid structures as phononic materials and its design optimization process have been presented in [12], where a limited number of continuously varying parameters define the geometry of a predefined cellular topology that deals with periodic structures of infinite size as well as demonstrate the validity of the results to finite systems.

Wave-wave interaction and its evolution in a continuum have been investigated [3], [13], [14] including theoretical study on the interaction of harmonic elastic plane waves in a cubically nonlinear material using the method of multiple scales [3]. The frequency vs. wave number dispersion relation of a one dimensional linear chain may be confined to the first Brillouin zone due to spatial periodicity [15]. Nonlinearities play an important and crucial role in the dynamics of this type of devices especially in small scale applications (e.g. Micro-Electro-Mechanical-Systems) [16], [17]. Hence understanding the influence of nonlinearities will allow possibilities of enhancing the material properties of these special group of materials.

Amplitude-dependent dispersion and bandgap behavior have been explored in several discrete periodic systems characterized by cubic nonlinearities by Narisetti et al. [6], where it was shown that the boundary of the dispersion curve may shift with amplitude in the presence of a single plane wave. Manktelow et al. [18] have recently extended the analysis in [6] to include the propagation of multiple harmonic plane waves that show the dispersion properties of discrete, periodic, cubically nonlinear systems. They presented a comparison between the multiple scales and Lindstedt-Poincare method for harmonic wave-wave

*corresponding author: Scientist/Research Engineer at Ford Motor Company, Research \& Advanced Engineering. email: smrutiranjan@gmail.com. 
interactions due to commensurate frequency ratios.

While these studies address cubic nonlinear periodic media for applications as metamaterials, quadratic nonlinearities have received limited attention for their wave propagations characteristics in periodic media. Our work studies the case of quadratic nonlinearity, which can represent situations of only quadratic nonlinearity, or where quadratic nonlinearity is dominant over cubic nonlinearity. If quadratic and cubic are equally present, we could not simply combine the quadratic only and cubic only phenomena since superposition does not apply to nonlinear systems. Hence, specific quadratic phenomena are worth understanding. This study follows up previous work done on single wave propagation in nonlinear chains with weak quadratic and cubic nonlinearities by Panigrahi et al. [19].

In this work, small amplitude waves propagating about an equilibrium configuration of a snap-through periodic chain are studied, while retaining the quadratic and cubic nonlinearity local to the equilibrium as shown in Section II. In Section III, a first-order multiple scales perturbation analysis is applied for low-amplitude oscillations that capture the quadratic effects. Interactions between two waves traveling through the chain and their stability analysis is shown. The dynamics of the slowly varying amplitude and phase in Section $\mathrm{V}$ shows the phase-drift and weakly phase-locking interactions and energy exchange between the waves along with emergent waveforms. In Section IV, the numerical simulations on the full chain is performed to validate the results obtained from the perturbation analysis. Finally, the concluding remarks are presented in Section S:Conclusion.

\section{Periodic Chain of Nonlinear Oscillators}

The wave behaviors in an infinite uniform nonlinear mass-spring chain (Figure 1) is studied. The mass-spring chain is arranged in a fashion such that each mass is separated by a distance $h$ from its nearest neighbor at equilibrium. It is assumed that all the masses are equal $\left(m_{j}=m\right)$ and only the nearest neighbors have direct effects on each other.

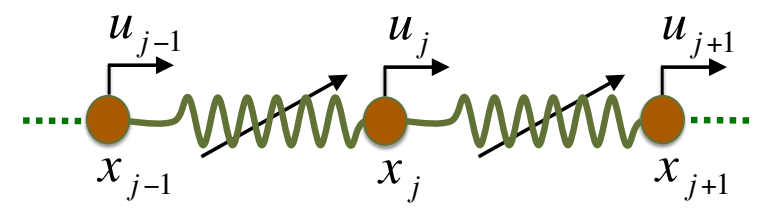

Fig. 1: Infinite mass chain. The unstretched position and displacement of mass $m_{j}$ are denoted by $x_{j}$ and $u_{j}$ respectively. The springs with unstretched length $h$, are cubic nonlinear with both quadratic and cubic nonlinearity.

Motivated by snap-through systems, in which either all springs are buckled to the first root, or all springs are stretched to the third root are considered. The masses can oscillate about this compressed or stretched equilibrium state. As such, a cubic expansion of the spring force relative to the equilibrium state leads to equations of motion (EOM) that can then be written as

$$
m \ddot{u}_{j}=\tilde{\alpha}\left[\left(u_{j+1}-u_{j}\right)-\left(u_{j}-u_{j-1}\right)\right]+\tilde{\beta}\left[\left(u_{j+1}-u_{j}\right)^{2}-\left(u_{j}-u_{j-1}\right)^{2}\right]+\tilde{\gamma}\left[\left(u_{j+1}-u_{j}\right)^{3}-\left(u_{j}-u_{j-1}\right)^{3}\right]
$$

for $j=\cdots,-2,-1,0,1,2, \cdots$. To simplify the equation the following coordinate transformation is applied:

$$
\epsilon z_{j}=\left(u_{j+1}-u_{j}\right)
$$

Letting $\alpha=\frac{\tilde{\alpha}}{m}, \beta=\frac{\tilde{\beta}}{m}, \gamma=\frac{\tilde{\gamma}}{m}$, the equations become

$$
\begin{gathered}
\ddot{u}_{j+1}=\epsilon \alpha\left(z_{j+1}-z_{j}\right)+\epsilon^{2} \beta\left(z_{j+1}^{2}-z_{j}^{2}\right)+\epsilon^{3} \gamma\left(z_{j+1}^{3}-z_{j}^{3}\right) \\
\ddot{u}_{j}=\epsilon \alpha\left(z_{j}-z_{j-1}\right)+\epsilon^{2} \beta\left(z_{j}^{2}-z_{j-1}^{2}\right)+\epsilon^{3} \gamma\left(z_{j}^{3}-z_{j-1}^{3}\right)
\end{gathered}
$$

Subtracting equation (4) from equation (3), the equation of motion in the $z$ coordinates is obtained as

$$
\ddot{z}_{j}=-\alpha\left(2 z_{j}-z_{j+1}-z_{j-1}\right)-\epsilon \beta\left(2 z_{j}^{2}-z_{j+1}^{2}-z_{j-1}^{2}\right)-\epsilon^{2} \gamma\left(2 z_{j}^{3}-z_{j+1}^{3}-z_{j-1}^{3}\right)
$$

When $\epsilon$ is small, the spring deformation near one of the stable equilibria is considered. With $\epsilon$ as a bookkeeping parameter, the quadratic effect is more dominant than the cubic in equation (5). With the small parameter $\epsilon$, these ordinary differential equations are analyzed by using the method of multiple scales next. In contrast to [19] which applied second-order perturbations to capture the quadratic effect, here a first-order perturbations is used, considering only the quadratic effect (i.e. no cubic elements). 


\section{PERTurbation AnAlysis of Interacting WAVES}

\section{A. Multiple Scales Analysis}

The wave equations are analyzed in this section using the method of multiple scales (MMS). A first-order expansion is considered, i.e. the only time scales of interest are $T_{0}=t$ and $T_{1}=\epsilon t$, where $T_{0}$ is the fast varying and $T_{1}$ is the slowly varying time scale. The strain variable $z_{j}(t)$ is expressed in multiple time scales as below,

$$
\begin{aligned}
& z_{j}(t)=z_{j_{0}}\left(T_{0}, T_{1}\right)+\epsilon z_{j_{1}}\left(T_{0}, T_{1}\right)+\cdots \\
& \frac{d}{d t}=D_{0}+\epsilon D_{1}+\epsilon^{2} D_{2}+\cdots \\
& \frac{d^{2}}{d t}=D_{0}^{2}+\epsilon\left(2 D_{0} D_{1}\right)+\epsilon^{2}\left(D_{1}^{2}+2 D_{0} D_{2}\right)+\cdots
\end{aligned}
$$

where $D_{i}=\frac{\partial}{\partial T_{i}}$. Substituting equation (6) into equation (5) yields

$$
D_{0}^{2} z_{j_{0}}+\epsilon\left[D_{0}^{2} z_{j_{1}}+2 D_{0} D_{1} z_{j_{0}}\right]=-\alpha\left[2 z_{j_{0}}-z_{j+1_{0}}-z_{j-1_{0}}\right]-\epsilon\left[\alpha\left(2 z_{j_{1}}-z_{j+1_{1}}-z_{j-1_{1}}\right)+\beta\left(2 z_{j_{0}}^{2}-z_{j+1_{0}}^{2}-z_{j-1_{0}}^{2}\right)\right]
$$

Equating like powers of $\epsilon$,

$$
\begin{gathered}
\epsilon^{0}: D_{0}^{2} z_{j_{0}}+\alpha\left(2 z_{j_{0}}-z_{j+1_{0}}-z_{j-1_{0}}\right)=0 \\
\epsilon^{1}: D_{0}^{2} z_{j_{1}}+\alpha\left(2 z_{j_{1}}-z_{j+1_{1}}-z_{j-1_{1}}\right)=-2 D_{0} D_{1} z_{j_{0}}-\beta\left(2 z_{j_{0}}^{2}-z_{j+1_{0}}^{2}-z_{j-1_{0}}^{2}\right)
\end{gathered}
$$

Assuming $x_{j \pm 1}=x_{j} \pm h$, and letting $x_{0}=0$ without loss of generality, it can be found that $x_{j}=j h$. The non-dimensional wavenumber is then $\mu=k h$, where $k$ is the wave number and $h$ is the spacial distance between the adjacent masses. Floquet-Bloch theory provides a strategy to analyze the wave dispersion behavior in a periodic structure. Hence we employ the Floquet-Bloch theory on a 1-D uniform chain to assume a wave-function solution to equation (8) obtained from the MMS applied to equation (5). Assuming a traveling wave solution in spatial and temporal terms with two harmonics to solve the $\epsilon^{0}$ equation (8), let

$$
z_{j_{0}}=y_{j_{A_{0}}}+y_{j_{B_{0}}}=A e^{i\left(\mu_{A_{0}} j-\omega_{A_{0}} T_{0}\right)}+B e^{i\left(\mu_{B_{0}} j-\omega_{B_{0}} T_{0}\right)}+\text { c.c. }
$$

where $y_{j_{A_{0}}}=A e^{i\left(\mu_{A_{0}} j-\omega_{A_{0}} T_{0}\right)}+$ c.c., and $y_{j_{B_{0}}}=B e^{i\left(\mu_{B_{0}} j-\omega_{B_{0}} T_{0}\right)}+$ c.c. are the waves A and B respectively, and c.c. represents the complex conjugates of all preceding terms. $\mu_{A_{0}}, \mu_{B_{0}}$ are the non-dimensional wave numbers and $\omega_{A_{0}}, \omega_{B_{0}}$ are

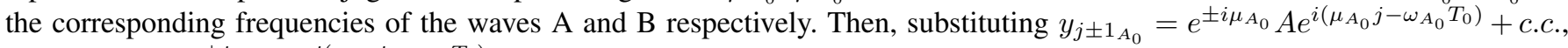
and $y_{j \pm 1_{B_{0}}}=e^{ \pm i \mu_{B_{0}} B e^{i\left(\mu_{B_{0}} j-\omega_{B_{0}} T_{0}\right)}}+c . c$. , into equation (8), the linearized form of the dispersion relation is found as

$$
\begin{aligned}
& \omega_{A_{0}}^{2}=2 \alpha\left(1-\cos \mu_{A_{0}}\right) \\
& \omega_{B_{0}}^{2}=2 \alpha\left(1-\cos \mu_{B_{0}}\right)
\end{aligned}
$$

Hence, letting $A\left(T_{1}\right)=\frac{1}{2} a\left(T_{1}\right) e^{-i \theta_{A}\left(T_{1}\right)}$, and $B\left(T_{1}\right)=\frac{1}{2} b\left(T_{1}\right) e^{-i \theta_{B}\left(T_{1}\right)}$, the solution to $\epsilon^{0}$ equation becomes

$$
z_{j_{0}}=\frac{1}{2} a e^{i\left(\mu_{A_{0}} j-\omega_{A_{0}} T_{0}-\theta_{A}\right)}+\frac{1}{2} b e^{i\left(\mu_{B_{0}} j-\omega_{B_{0}} T_{0}-\theta_{B}\right)}+c . c .
$$

For non-zero amplitudes $a$ and $b$, the relationship between $\theta_{A}$ and $\theta_{B}$ determines whether the response $z_{j_{0}}$ in equation (12), with phases $\left(\mu_{A_{0}} j-\omega_{A_{0}} T_{0}-\theta_{A}\right)$, and $\left(\mu_{B_{0}} j-\omega_{B_{0}} T_{0}-\theta_{B}\right)$, is in continuous drift with difference $\theta=\left(\mu_{A_{0}}-\mu_{B_{0}}\right) j-$ $\left(\omega_{A_{0}}-\omega_{B_{0}}\right) T_{0}-\left(\theta_{A}-\theta_{B}\right)$, known as phase drift, or becomes phase locked. Using this solution of $z_{j_{0}}$ in equation (9) results in the following equation:

$$
\begin{aligned}
D_{0}^{2} z_{j_{1}}+ & \alpha\left(2 z_{j_{1}}-z_{j+1_{1}}-z_{j-1_{1}}\right) \\
= & i \omega_{A_{0}}\left(a^{\prime}-i a \theta_{A}^{\prime}\right) e^{i \tilde{\theta}_{A}}+i \omega_{B_{0}}\left(b^{\prime}-i b \theta_{B}^{\prime}\right) e^{i \tilde{\theta}_{B}}+c . c . \\
- & \frac{\beta}{4}\left[2\left\{\left[a^{2} e^{2 i \tilde{\theta}_{A}}+b^{2} e^{2 i \tilde{\theta}_{B}}+2 a b e^{i\left(\tilde{\theta}_{A}+\tilde{\theta}_{B}\right)}\right]+\left[a^{2}+b^{2}+2 a b e^{i\left(\tilde{\theta}_{B}-\tilde{\theta}_{A}\right)}\right]+c . c .\right\}\right. \\
& -\left\{\left[a^{2} e^{2 i \mu_{A_{0}}} e^{2 i \tilde{\theta}_{A}}+b^{2} e^{2 i \mu_{B_{0}}} e^{2 i \tilde{\theta}_{B}}+2 a b e^{i\left(\mu_{A_{0}}+\mu_{B_{0}}\right)} e^{i\left(\tilde{\theta}_{A}+\tilde{\theta}_{B}\right)}\right]+\left[a^{2}+b^{2}+2 a b e^{i\left(\mu_{B_{0}}-\mu_{A_{0}}\right)} e^{i\left(\tilde{\theta}_{B}-\tilde{\theta}_{A}\right)}\right]+c . c .\right\} \\
& \left.-\left\{\left[a^{2} e^{-2 i \mu_{A_{0}}} e^{2 i \tilde{\theta}_{A}}+b^{2} e^{-2 i \mu_{B_{0}}} e^{2 i \tilde{\theta}_{B}}+2 a b e^{-i\left(\mu_{A_{0}}+\mu_{B_{0}}\right)} e^{i\left(\tilde{\theta}_{A}+\tilde{\theta}_{B}\right)}\right]+\left[a^{2}+b^{2}+2 a b e^{-i\left(\mu_{B_{0}}-\mu_{A_{0}}\right)} e^{i\left(\tilde{\theta}_{B}-\tilde{\theta}_{A}\right)}\right]+c . c .\right\}\right] \\
= & i \omega_{A_{0}}\left(a^{\prime}-i a \theta_{A}^{\prime}\right) e^{i \tilde{\theta}_{A}}+i \omega_{B_{0}}\left(b^{\prime}-i b \theta_{B}^{\prime}\right) e^{i \tilde{\theta}_{B}}+c . c .-\frac{\beta}{2}\left[a^{2}\left[1-\cos \left(2 \mu_{A_{0}}\right)\right] e^{2 i \tilde{\theta}_{A}}+b^{2}\left[1-\cos \left(2 \mu_{B_{0}}\right)\right] e^{2 i \tilde{\theta}_{B}}\right. \\
& \left.+2 a b\left[1-\cos \left(\mu_{B_{0}}+\mu_{A_{0}}\right)\right] e^{i\left(\tilde{\theta}_{B}+\tilde{\theta}_{A}\right)}+2 a b\left[1-\cos \left(\mu_{B_{0}}-\mu_{A_{0}}\right)\right] e^{i\left(\tilde{\theta}_{B}-\tilde{\theta}_{A}\right)}+c . c .\right]
\end{aligned}
$$


where, $\tilde{\theta}_{A}=\mu_{A_{0}} j-\omega_{A_{0}} T_{0}-\theta_{A}$, and $\tilde{\theta}_{B}=\mu_{B_{0}} j-\omega_{B_{0}} T_{0}-\theta_{B}$.

The secular terms are the coefficients of $e^{i\left(\mu_{A_{0}} j-\omega_{A_{0}} T_{0}\right)}$ and $e^{i\left(\mu_{B_{0}} j-\omega_{B_{0}} T_{0}\right)}$. Primary resonance occurs when $\omega_{A_{0}} \approx \omega_{B_{0}}$ and $\mu_{A_{0}} \approx \mu_{B_{0}}$, such that the solvability conditions are

$$
\begin{array}{ll}
\text { Wave-A : } & i \omega_{A}\left(a^{\prime}-i \theta_{A}^{\prime} a\right)=0 \\
\text { Wave-B : } \quad i \omega_{B}\left(b^{\prime}-i \theta_{B}^{\prime} b\right)=0
\end{array}
$$

which simplifies to the following equations

$$
\begin{aligned}
\omega_{A} a^{\prime} & =0 \\
\omega_{A} a \theta_{A}^{\prime} & =0 \\
\omega_{B} b^{\prime} & =0 \\
\omega_{B} b \theta_{B}^{\prime} & =0
\end{aligned}
$$

For primary resonance in the above equation, the amplitudes and the phases of both waves A, and B become independent of the slow time scale $T_{1}$, and hence do not contain any slow scale dynamics. In that case one would require higher-order terms to show the effects of the primary resonances.

However, there is interaction between the two waves resulting in super- and sub-harmonic resonances. Due to the arbitrary naming convention of the $\mathrm{A}$ and $\mathrm{B}$ waves, the secular terms arising from super- and sub-harmonics are identical. The assumptions $\omega_{B_{0}} \cong 2 \omega_{A_{0}}$ and $\mu_{B_{0}} \cong 2 \mu_{A_{0}}$ are made for resonance analysis. This inequality is then converted into the following relations by introducing the detuning parameters $\sigma_{\omega}$ and $\sigma_{\mu}$ as

$$
\begin{aligned}
& \omega_{B_{0}}=2 \omega_{A_{0}}+\epsilon \sigma_{\omega} \\
& \mu_{B_{0}}=2 \mu_{A_{0}}+\epsilon \sigma_{\mu}
\end{aligned}
$$

where $\sigma_{\omega}$, and $\sigma_{\mu}$ are of order one. Note that $\sigma_{\omega}$ and $\sigma_{\mu}$ are not independent of each other. For example, given $\sigma_{\omega}$ and $\omega_{A}$, $\omega_{B}$ can be computed from equation (16)(a) and $\mu_{A}$ from equation (11). Then $\omega_{B}$ defines $\mu_{B}$ in equation (11). Finally $\sigma_{\mu}$ is obtained from equation (16)(b).

For wave $\mathrm{A}$, the secular terms (coefficients of $\left.e^{i\left(\mu_{A_{0}} j-\omega_{A_{0}} T_{0}\right)}\right)$ are

$$
i \omega_{A_{0}}\left(a^{\prime}-i a \theta_{A}^{\prime}\right) e^{-i \theta_{A}}=\beta a b\left[1-\cos \left(\mu_{B_{0}}-\mu_{A_{0}}\right)\right] e^{-i\left(\theta_{B}-\theta_{A}\right)} e^{i \epsilon\left(\sigma_{\mu} j-\sigma_{\omega} T_{0}\right)}
$$

and for wave $\mathrm{B}$, the secular terms (coefficients of $\left.e^{i\left(\mu_{B_{0}} j-\omega_{B_{0}} T_{0}\right)}\right)$ are

$$
i \omega_{B_{0}}\left(b^{\prime}-i b \theta_{B}^{\prime}\right) e^{-i \theta_{B}}=\frac{\beta}{2} a^{2}\left[1-\cos \left(2 \mu_{A_{0}}\right)\right] e^{-i 2 \theta_{A}} e^{-i \epsilon\left(\sigma_{\mu} j-\sigma_{\omega} T_{0}\right)}
$$

Equating the real and imaginary parts the equations for the slow scale dynamics leads to

$$
\begin{aligned}
a^{\prime} & =\frac{\beta a b}{\omega_{A_{0}}}\left[1-\cos \left(\mu_{B_{0}}-\mu_{A_{0}}\right)\right] \sin \phi \\
b^{\prime} & =-\frac{\beta a^{2}}{2 \omega_{B_{0}}}\left[1-\cos \left(2 \mu_{A_{0}}\right)\right] \sin \phi \\
\theta_{A}^{\prime} & =\frac{\beta b}{\omega_{A_{0}}}\left[1-\cos \left(\mu_{B_{0}}-\mu_{A_{0}}\right)\right] \cos \phi \\
\theta_{B}^{\prime} & =\frac{\beta a^{2}}{2 b \omega_{B_{0}}}\left[1-\cos \left(2 \mu_{A_{0}}\right)\right] \cos \phi
\end{aligned}
$$

where ()$^{\prime}$ denotes the derivative with respect to slowly varying time scale $T_{1}$. Similar equations are seen, for example, with the swinging spring [20]. In the above equations $\phi$ is a function of the phases of the waves $A$ and $B$, the detuning parameters $\sigma_{\omega}$ and $\sigma_{\mu}$, and the time scale $T_{1}$ as

$$
\begin{aligned}
\phi & =\left(2 \theta_{A}-\theta_{B}\right)+\epsilon \sigma_{\mu} j-\sigma_{\omega} T_{1} \\
\phi^{\prime} & =\left(2 \theta_{A}^{\prime}-\theta_{B}^{\prime}\right)-\sigma_{\omega}
\end{aligned}
$$

Using equation (20) in equation (19), the 4-D phase space can be reduced to three as

$$
\begin{aligned}
a^{\prime} & =\frac{\alpha_{1}}{\omega_{A_{0}}} a b \sin \phi \\
b^{\prime} & =-\frac{\alpha_{2}}{\omega_{B_{0}}} a^{2} \sin \phi \\
\phi^{\prime} & =-\sigma_{\omega}+\left[\frac{2 \alpha_{1}}{\omega_{A_{0}}} b^{2}-\frac{\alpha_{2}}{\omega_{B_{0}}} a^{2}\right] \frac{1}{b} \cos \phi
\end{aligned}
$$


where $\alpha_{1}=\beta\left[1-\cos \left(\mu_{B_{0}}-\mu_{A_{0}}\right)\right]$ and $\alpha_{2}=\frac{\beta}{2}\left[1-\cos \left(2 \mu_{A_{0}}\right)\right]$ are functions of nonlinearity coefficient $\beta$ and the nondimensional wave numbers of waves $\mathrm{A}$ and $\mathrm{B}$, and are independent of the amplitudes and phases. For non-zero $a, b, a^{\prime}$, and $b^{\prime}$, dividing the two amplitude equations and letting $\frac{d a}{d T_{1}} / \frac{d b}{d T_{1}}=\frac{d a}{d b}$, it is found that

$$
\frac{d a}{d b}=-\frac{\omega_{B_{0}}}{\omega_{A_{0}}} \frac{\alpha_{1} b}{\alpha_{2} a}=-\frac{1}{r} \frac{b}{a}
$$

where $r=\frac{\alpha_{2} \omega_{A_{0}}}{\alpha_{1} \omega_{B_{0}}}=\frac{\sin ^{2}\left(\mu_{A_{0}}\right) \sin \left(\frac{\mu_{A_{0}}}{2}\right)}{2 \sin ^{2}\left(\frac{\mu_{A_{0}}}{2}+\frac{\epsilon \sigma_{\mu}}{2}\right) \sin \left(\mu_{A_{0}}+\frac{\epsilon \sigma_{\mu}}{2}\right)}$. Cross multiplying and integrating the above equation results in

$$
r a^{2}+b^{2}=E
$$

where $E$ is the integration constant and can be considered as the characteristic energy of the system.

Based on equation (19), the strength $\beta$ of the quadratic nonlinearity affects the time rate at which the energy is exchanged. For different energy states the non-zero amplitudes of these waves are confined to corresponding concentric ellipses in the $a-b$ amplitude space for a given $r$ which depends on $\mu_{A_{0}}$ and the detuning parameter $\sigma_{\mu}$. However, the shape of this ellipse can change depending on parameters such as the detuning parameter. When $a, b, a^{\prime}$, and $b^{\prime}$ all are non-zero, the amplitudes oscillate on an ellipse in the $a-b$ plane for a particular energy state. When $a^{\prime}$ and $b^{\prime}$ are zero, the phase is either zero or $n \pi$ which also represents the steady state.

Using equation (23) in equation (21), the dimension of phase orbits can be reduced from three to two. This means that there exists a 3-D phase space ( $a, b, \phi$ as the states), but the motion is constrained/confined to a 2-D surface defined by the ellipses of $a, b$ as in equation (23). Hence the reduced amplitude-phase equations in 2-D are, for example in terms of $b$,

$$
\begin{aligned}
b^{\prime} & =-\frac{\alpha_{1}}{\omega_{A_{0}}}\left[E-b^{2}\right] \sin \phi \\
\phi^{\prime} & =-\sigma_{\omega}-\frac{\alpha_{1}}{\omega_{A_{0}} b}\left[E-3 b^{2}\right] \cos \phi
\end{aligned}
$$

In order to solve for the the amplitude and phase, ratio of the phase and amplitude equations in (24) is obtained, to get further reduced equation in $1-\mathrm{D}$, as

$$
\frac{d \phi}{d b}=\frac{g_{1}(b) \cos \phi}{\sin \phi}-\frac{g_{2}(b)}{\sin \phi}
$$

where $g_{1}(b)=\frac{E-3 b^{2}}{b\left(E-b^{2}\right)}$, and $g_{2}(b)=-\frac{\omega_{A_{0}} \sigma_{\omega}}{\alpha_{1}\left(E-b^{2}\right)}$.

Letting $\cos \phi=p$, and $(-\sin \phi) d \phi=d p$, a first-order linear differential equation of the form

$$
\frac{d p(b)}{d b}+g_{1}(b) p(b)=g_{2}(b)
$$

is obtained, in which $b$ is the independent variable and $p$ is the dependent variable. The relationship between the amplitude $b$ and phase $\phi$ can then be written as

$$
p(b)=\cos \phi=e^{-\int g_{1}(b) d b}\left[\int g_{2}(b) e^{\int g_{1}(b) d b} d b+c_{1}\right]
$$

where $c_{1}$ is an integration constant, and the integrating factor is $e^{\int g_{1}(b) d b}=b\left(E-b^{2}\right)$.

Introducing the integrating factor results in $\int g_{2}(b) e^{\int g_{1}(b) d b} d b=-\frac{\omega_{A_{0}} \sigma_{\omega}}{2 \alpha_{1}} b^{2}$. The relationship between the amplitude $b$ and the phase $\phi$ is therefore obtained from the solution to our differential equation as

$$
\cos \phi=\frac{1}{b\left(E-b^{2}\right)}\left[-\frac{\omega_{A_{0}} \sigma_{\omega}}{2 \alpha_{1}} b^{2}+c_{1}\right]
$$

Constant $c_{1}$ depends on the value of $\phi$ at a specified value of $b$.

\section{B. Stability Analysis}

There are two set of fixed points corresponding to the slow flow equations. For the first set of fixed points, substituting $b^{\prime}=\phi^{\prime}=0$ into the 2-D phase space equation (24) results in the following two equations:

$$
\begin{aligned}
b_{0}^{2} & =E \\
\cos \phi_{0} & = \pm \frac{\sigma_{\omega} \omega_{A_{0}}}{2 \alpha_{1} \sqrt{E}}
\end{aligned}
$$

which exists if $\left|\sigma_{\omega} \omega_{A_{0}}\right| \leq 2 \alpha_{1} \sqrt{E}$. From equation (23), this fixed point indicates $a_{0}=0$, and thus represents a single harmonic wave. 


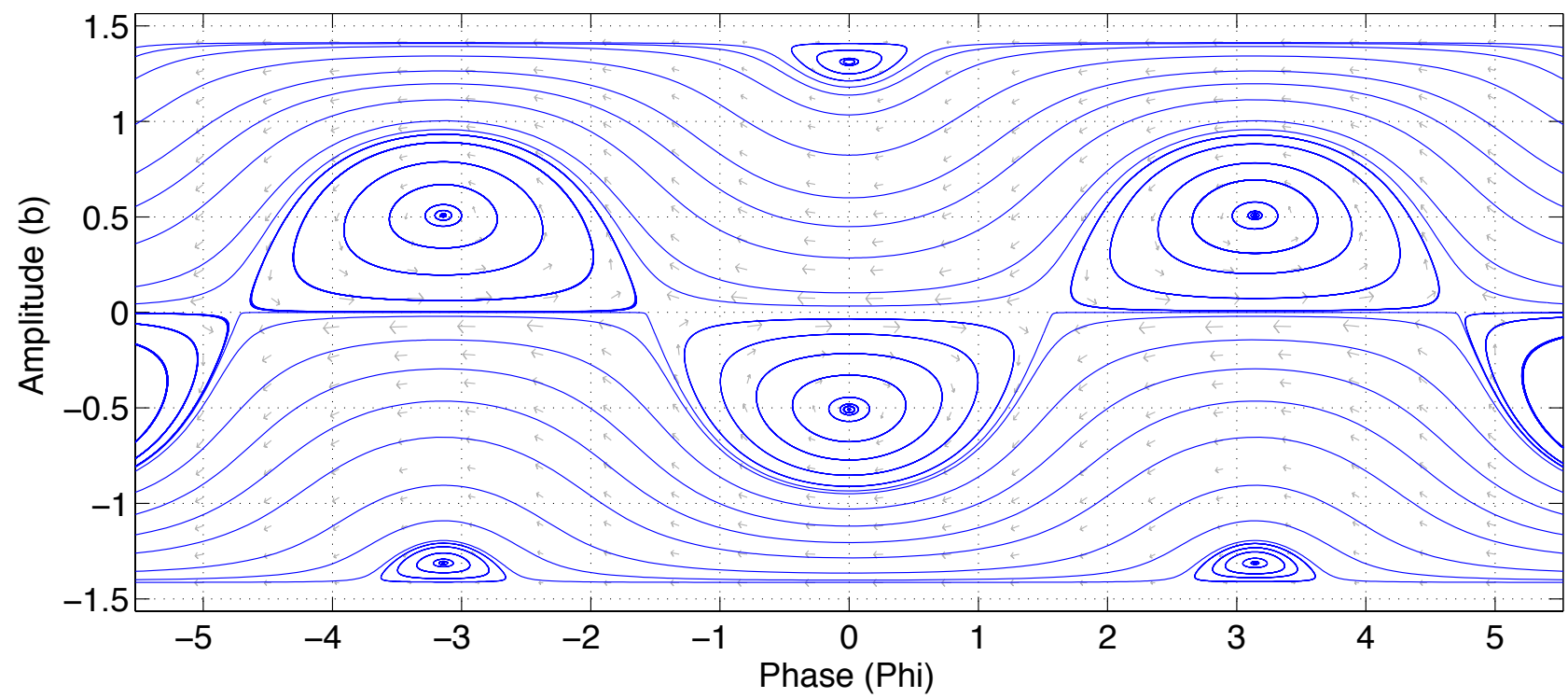

Fig. 2: This is the phase plot of the slow scale dynamics corresponding to equation (24) of a multi-wave propagation in an infinite nonlinear chain with weak quadratic nonlinearity. The horizontal axis represents the phase $\phi$ and the vertical axis represents the amplitude $b$. The figure corresponds to wave number $\mu_{A_{0}}=0.5, \epsilon=0.1, E=2$, and $\sigma_{\omega}=1$, and $\beta=\alpha=1$. The frequency $\omega_{A_{0}}$ can be found by substituting the values of $\mu_{A_{0}}$ and $\alpha$ into equation (11) and is shown in Table 2 .

A second set of fixed points exist for non-zero equilibrium amplitudes and corresponds to

$$
\begin{aligned}
\sin \phi_{0} & =0 \\
b_{0}^{2}-\tilde{\sigma} b_{0}-\tilde{E} & =0
\end{aligned}
$$

where $\tilde{\sigma}=( \pm) \frac{\omega_{A_{0}} \sigma_{\omega}}{3 \alpha_{1}}$, and $\tilde{E}=\frac{E}{3}$. The above equation is a quadratic equation for the fixed point $b_{0}$ that can be solved to get the equilibrium amplitude as

$$
b_{0}=\frac{1}{2}\left[\tilde{\sigma} \pm \sqrt{\tilde{\sigma}^{2}+4 \tilde{E}}\right]
$$

For the stability of these steady-state amplitude and phases, the Jacobian of the equation (24) is first computed as

$$
\mathbf{J}=\left[\begin{array}{cc}
\frac{2 \alpha_{1}}{\omega_{A_{0}}} b_{0} \sin \phi_{0} & -\frac{\alpha_{1}}{\omega_{A_{0}}}\left(E-b_{0}^{2}\right) \cos \phi_{0} \\
\frac{\alpha_{1}}{\omega_{A_{0}} b_{0}^{2}}\left(E+3 b_{0}^{2}\right) \cos \phi_{0} & \frac{\alpha_{1}}{\omega_{A_{0}} b_{0}}\left(E-3 b_{0}^{2}\right) \sin \phi_{0}
\end{array}\right]
$$

At the first set of fixed points from equation (29), the Jacobian becomes

$$
\mathbf{J}_{\mathbf{1}}=\left[\begin{array}{cc}
\sigma_{\omega} \tan \phi_{0} & 0 \\
\frac{4 \alpha_{1}}{\omega_{A_{0}}} \cos \phi_{0} & -\sigma_{\omega} \tan \phi_{0}
\end{array}\right]
$$

and the corresponding eigenvalues are

$$
\lambda_{1,2}= \pm \sigma_{\omega} \tan \phi_{0}
$$

Therefore these fixed points are unstable saddle points.

The Jacobian of the second set of fixed points for $\sin \phi_{0}=0$ is

$$
\mathbf{J}_{\mathbf{2}}=\left[\begin{array}{cc}
0 & -\frac{\alpha_{1}}{\omega_{A_{0}}}\left(E-b_{0}^{2}\right)( \pm 1) \\
\frac{\alpha_{1}}{\omega_{A_{0}} b_{0}^{2}}\left(E+3 b_{0}^{2}\right)( \pm 1) & 0
\end{array}\right]
$$

and the corresponding eigenvalues are

$$
\lambda^{2}=-\frac{\alpha_{1}^{2}}{\omega_{A_{0}}^{2} b_{0}^{2}}\left(E-b_{0}^{2}\right)\left(E+3 b_{0}^{2}\right)<0
$$

Hence these fixed points are centers, and turn out to be neutrally stable fixed points.

\section{Wave-Wave Waveforms}

The wave-wave interactions between two waves result in exchange of energy between the two waves that can be seen in the simulations of the resultant waveform. Given solutions and behavior of the state variables $(a, b, \phi)$ we can reconstruct the 


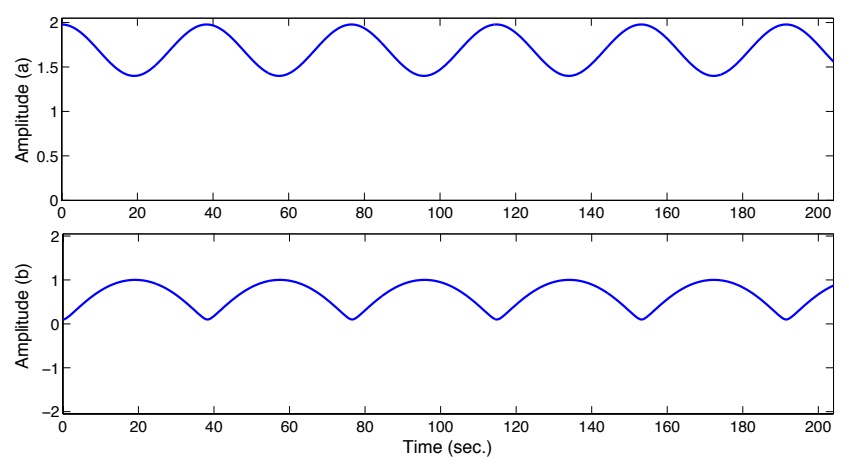

(a)

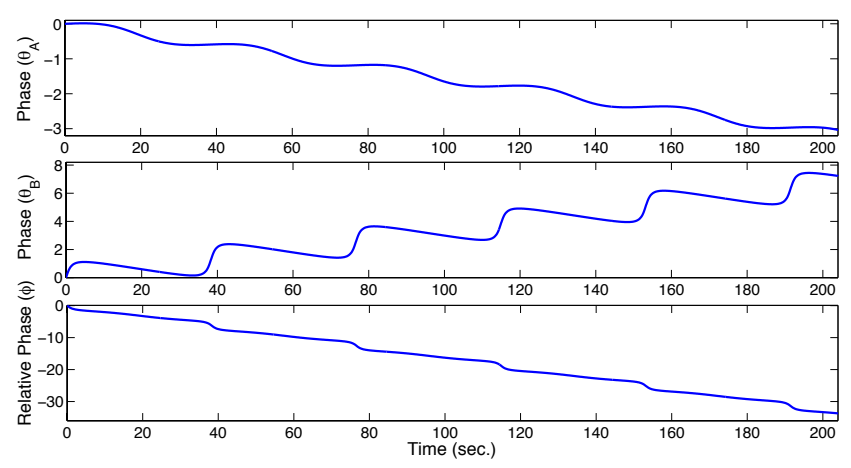

(b)

Fig. 3: (a) Time evolution of slowly varying (a) amplitudes and (b) phase for the initial conditions $b_{0}=0.1$ and $\phi_{0}=0$.

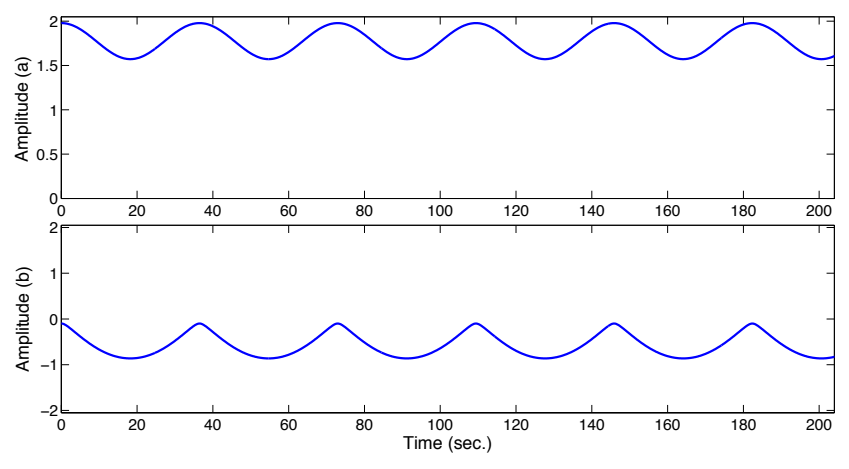

(a)

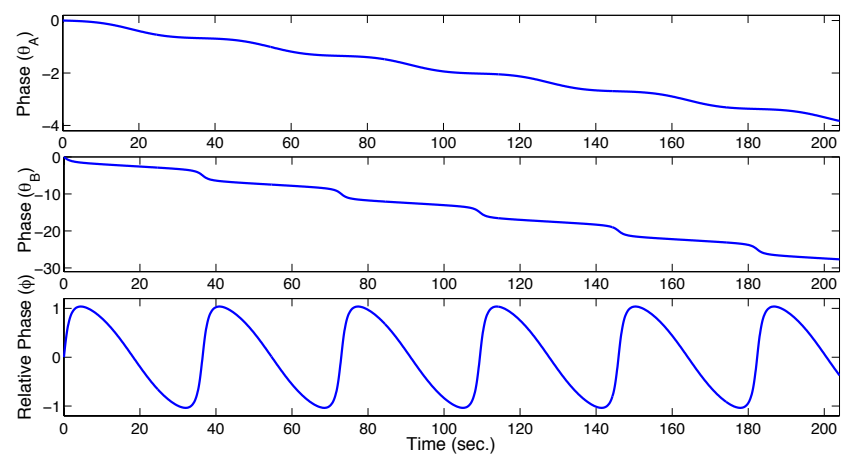

(b)

Fig. 4: (a) Time evolution of slowly varying (a) amplitudes and (b) phase for the initial conditions $b_{0}=-0.1$ and $\phi_{0}=0$.

wave-wave waveform as follows. From equation (12), the form of the leading order wave in strain coordinate is

$$
z_{j_{0}}=a \cos \left(\mu_{A_{0}} j-\omega_{A_{0}} T_{0}-\theta_{A}\right)+b \cos \left(\mu_{B_{0}} j-\omega_{B_{0}} T_{0}-\theta_{B}\right)
$$

Here $\omega_{B_{0}}=2 \omega_{A_{0}}+\epsilon \sigma_{\omega}, \mu_{B_{0}}=2 \mu_{A_{0}}+\epsilon \sigma_{\mu}$, and $\theta_{B}=-\phi+2 \theta_{A}+\epsilon \sigma_{\mu} j-\sigma_{\omega} T_{1}$. Substituting these in equation (37)

$$
\begin{aligned}
z_{j_{0}} & =a \cos \left(\mu_{A_{0}} j-\omega_{A_{0}} T_{0}-\theta_{A}\right)+b \cos \left(2\left(\mu_{A_{0}} j-\omega_{A_{0}} T_{0}-\theta_{A}\right)+\phi\right) \\
& =a \cos \left[\mu_{A_{0}}\left(j-\frac{\omega_{A_{0}}}{\mu_{A_{0}}} T_{0}-\frac{\theta_{A}\left(T_{1}\right)}{\mu_{A_{0}}}\right)\right]+b \cos \left[2 \mu_{A_{0}}\left(j-\frac{\omega_{A_{0}}}{\mu_{A_{0}}} T_{0}-\frac{\theta_{A}\left(T_{1}\right)}{\mu_{A_{0}}}\right)+\phi\left(T_{1}\right)\right]
\end{aligned}
$$

Therefore, the speed of the resultant wave is $c=\frac{d}{d t}\left[\frac{\omega_{A_{0}}}{\mu_{A_{0}}} T_{0}-\frac{\theta_{A}\left(T_{1}\right)}{\mu_{A_{0}}}\right]=\left[\frac{\partial}{\partial T_{0}}+\epsilon \frac{\partial}{\partial T_{1}}\right]\left[\frac{\omega_{A_{0}}}{\mu_{A_{0}}} T_{0}-\frac{\theta_{A}\left(T_{1}\right)}{\mu_{A_{0}}}\right]=\left[\frac{\omega_{A_{0}}}{\mu_{A_{0}}}-\epsilon \frac{\theta_{A}^{\prime}\left(T_{1}\right)}{\mu_{A_{0}}}\right]$ and the phase fluctuation is $\phi\left(T_{1}\right)$. The amplitudes $a$ and $b$ of Waves A and B respectively can fluctuate through the $(b, \phi)$ state-space dynamics and with an energy exchange according to the energy ellipse relations shown in equation (23).

\section{State-Space Dynamics of Interacting WaVes}

In this section we look at the dynamics of the slowly varying state variables $(a, b, \phi)$ and make interpretations about the interacting waves.

A $(b, \phi)$ phase portrait is shown in Figure 2. The phase plot is symmetric about the $b$-axis and the phase plot is periodic in $\phi$ with period $2 \pi$. This can be shown by letting $h(b, \phi)=\frac{d \phi}{d b}$ as defined in equation (25). It is observed that $h(b,-\phi)=-h(b, \phi)$ and $h(b, 2 \pi+\phi)=h(b, \phi)$. Within a period of $2 \pi$, the fixed points are at $\phi_{0}=0, \phi_{0}= \pm \pi$, and $\cos \phi_{0}= \pm \frac{\sigma_{\omega} \omega_{A_{0}}}{2 \alpha_{1} \sqrt{E}}$. The eigenvalues calculated in equations (34) and (36) at these fixed points prove that the fixed points are either centers or saddles and that the saddles are connected in the phase plot. This results into situations where there is phase-drift outside the separatrix and weak phase-locking inside the separatrix. Weak phase locking occurs as periodic orbits centered about equilibrium in the $b$ - $\phi$ state space. The fixed points in the $(b, \phi)$ space indicate strong phase locking.

Also observe that the phase diagram is symmetric about the $\phi$ axis. For $\mu_{A_{0}}=0.5$, there exists two centers for $\phi=0$ and another two centers for $\phi=\pi$. Connecting saddles are present in between these centers. The phase drifts are seen outside the separatrix.

Depending on parameters and the energy states, the interactions (i.e. $a, b, \phi)$ are periodic or whirling, such that the combined propagating waves are quasi-periodic with both phase-drifts and weakly phase-locking behavior. The time evolution of the amplitudes and phases of the two drifting waves are shown in Figures 3, and the two weakly locked waves in Figures 4. 


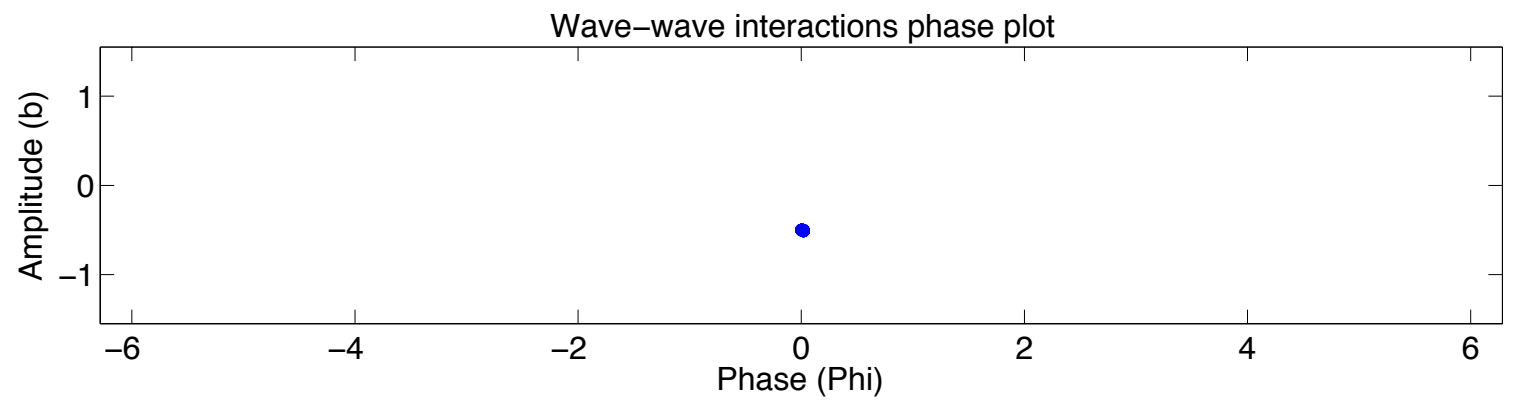

Wave-wave interactions in traveling waveform

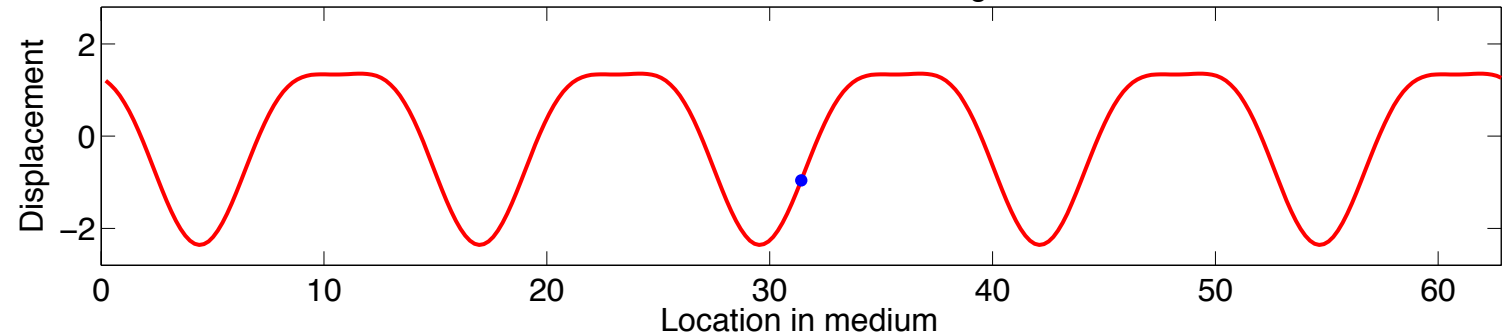

Fig. 5: This plot shows the phase plot corresponding to equation (24) and a snapshot of the time evolution of wave propagation in a chain at a stable equilibrium position i.e. a phase-locked waveform. The blue dot on the top plot represents the location of the fixed point at $\phi_{0}=0, b_{0}=-0.5$ in the phase trajectories. The time evolution snapshot on the bottom plot corresponds to the fixed point on the top plot. The shape of the waveform at a fixed point remains unchanged over time.
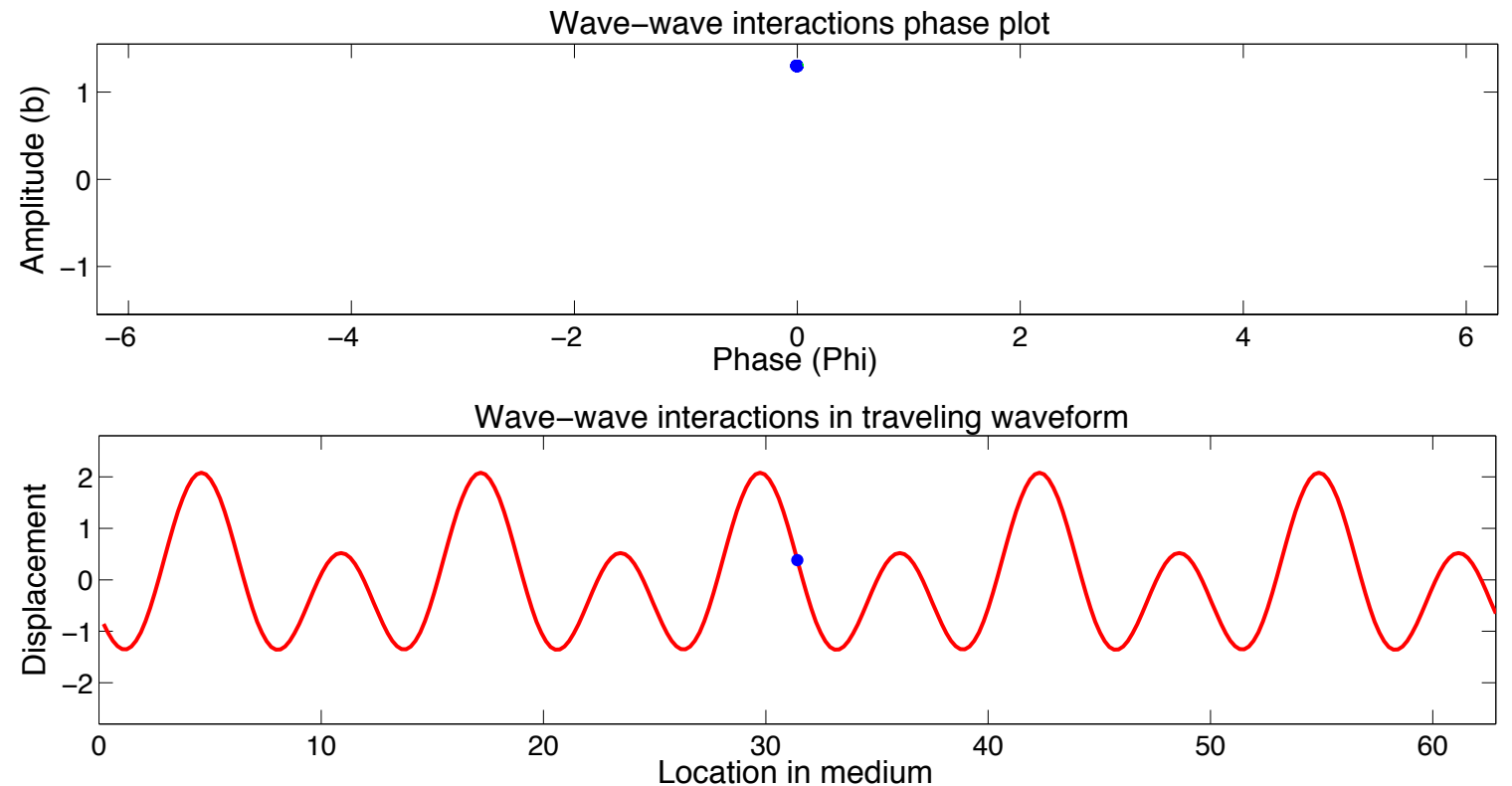

Fig. 6: This plot shows the phase plot corresponding to equation (24) and a snapshot of the time evolution of wave propagation in a chain at a fixed point in $(b, \phi)$ i.e. a phase-locked waveform. The blue dot on the top plot represents the location of the fixed point at $\phi_{0}=0, b_{0}=1.3$ in the phase space. The time evolution snapshot on the bottom plot corresponds to the fixed point on the top plot. The shape of the wave form at this fixed point remains unchanged over time.

The approximated dynamics in the chain can be reconstructed using equation (12) with the use of the $(b, \phi)$ phase-space dynamics, the energy ellipse of equation (23), and the phase relationships in equation (20).

\section{A. Wave-Wave Interactions Examples}

Some example cases are illustrated in the form of phase-space and snapshots of the waveforms in space. Figures 5 to 9 show phase diagrams for $b$ and $\phi$ and a snapshot of a sample traveling wave in original coordinates, for a given set of parameters. The slow-flow equations (24) were solved for $\mu_{A_{0}}=0.5$. The parameters assumed in the simulation of the autonomous phase space equations are shown in Table I and the derived parameter values shown in Table II. The initial conditions for strongly phase-locked, weakly phase-locked, and phase-drift examples shown in Figures 5 to 9 are tabulated in Table III. Assuming the 

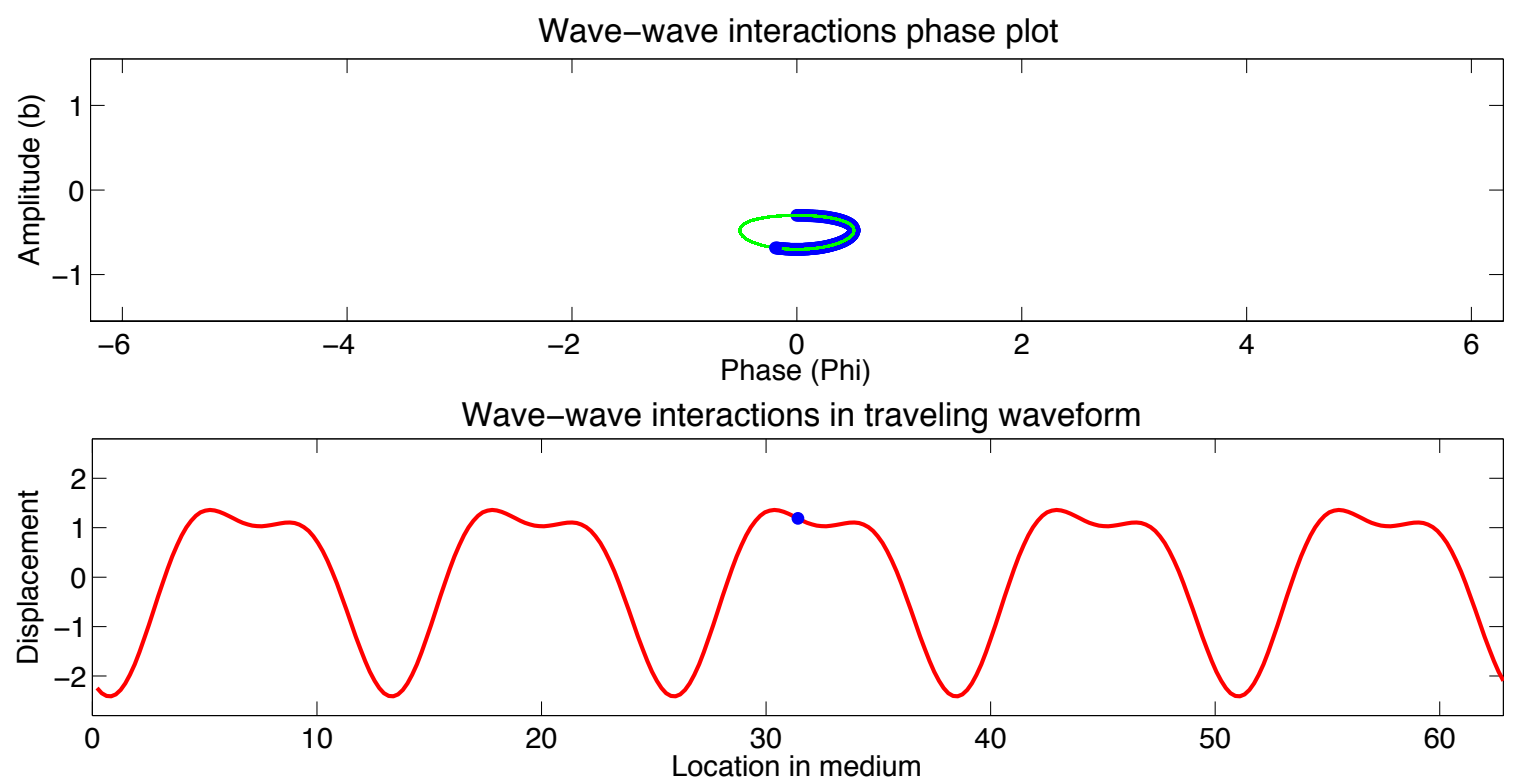

Fig. 7: This plot shows the phase plot corresponding to equation (24) and a snapshot of the time evolution of wave propagation in a chain in weakly phase locked behavior for initial conditions $\phi_{0}=0$, and $b_{0}=-0.3$. The green line on the top plot represents the phase trajectories for one weakly phase locked condition. The time evolution snapshot on the bottom plot corresponds to a point on the thick blue-green curve on the top figure. The shape of the spatial wave would have changed in the next time step.
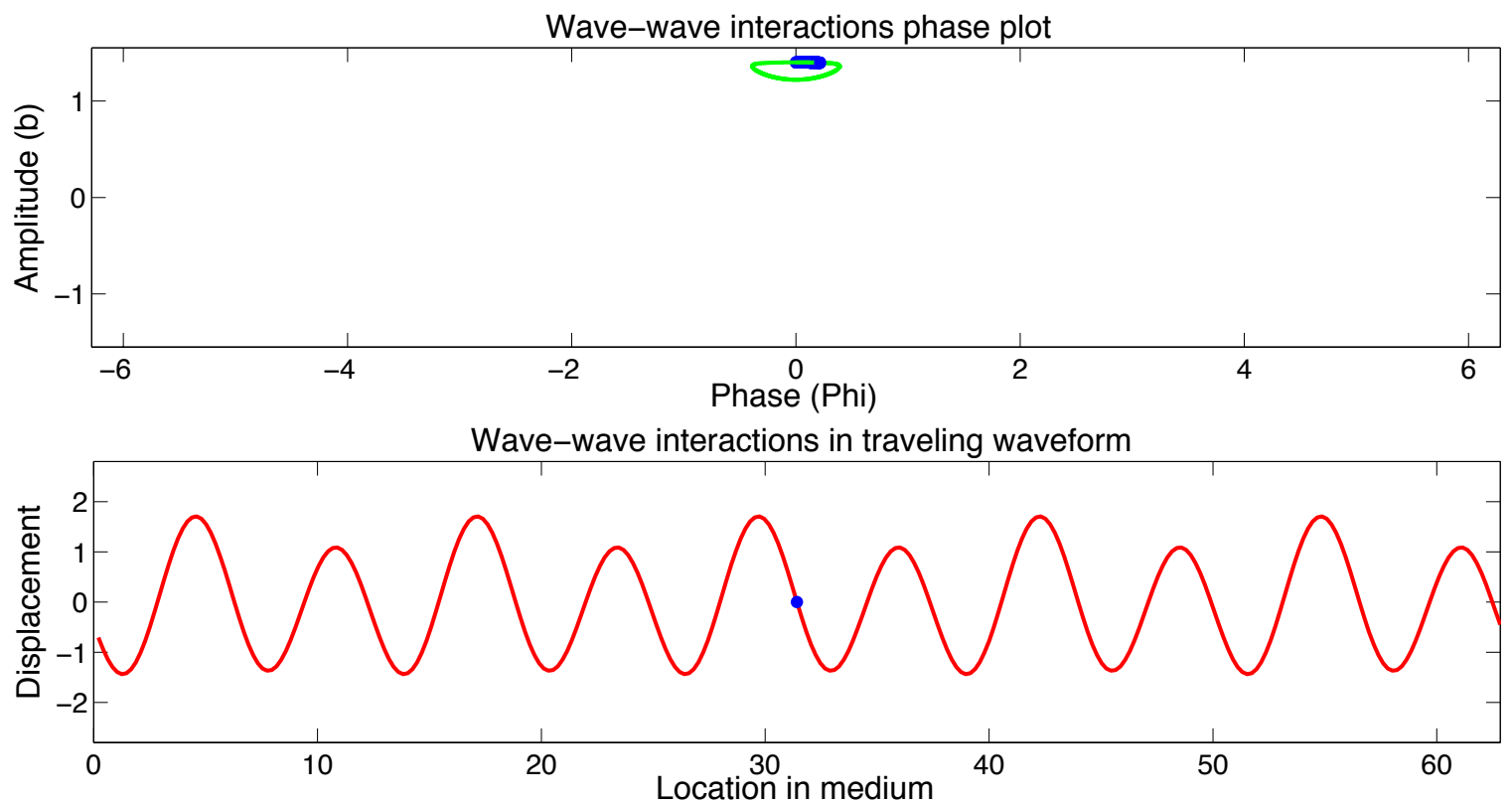

Fig. 8: This plot shows the phase plot corresponding to equation (24) and a snapshot of the time evolution of wave propagation in a chain in weakly phase locked behavior for initial conditions $\phi_{0}=0$, and $b_{0}=1.4$. The green line on the top plot represents a phase trajectory for one weakly phase locked condition. The time evolution snapshot on the bottom plot corresponds to a point on the thick blue-green curve on the top figure. The shape of the spatial wave would have changed in the next time step.

initial conditions $\phi_{0}, \theta_{A_{0}}$, and $b_{0}$, the other initial conditions in Table III are computed using the formulas $\theta_{B_{0}}=-\phi_{0}+2 \theta_{A_{0}}$, and $a_{0}=\sqrt{\frac{E-b_{0}^{2}}{r}}$.

Snapshots of the two fixed points, showing phase-locked waveforms, corresponding to $\phi_{0}=0$ are obtained from equation (31) and shown in Figure 5 for $b_{0}=-0.5$ and in Figure 6 for $b_{0}=1.3$. The complete simulation movies can be viewed from the attachments. Movie 1 corresponds to Figure 5 and Movie 2 corresponds to Figure 6.

From this analysis and from the phase plots in Figures 7 and 8 it can be realized that, for amplitudes and phases near the centers, the waves travel with a slight variation in shape due to the weak phase-locking. Amplitudes and phases away from 

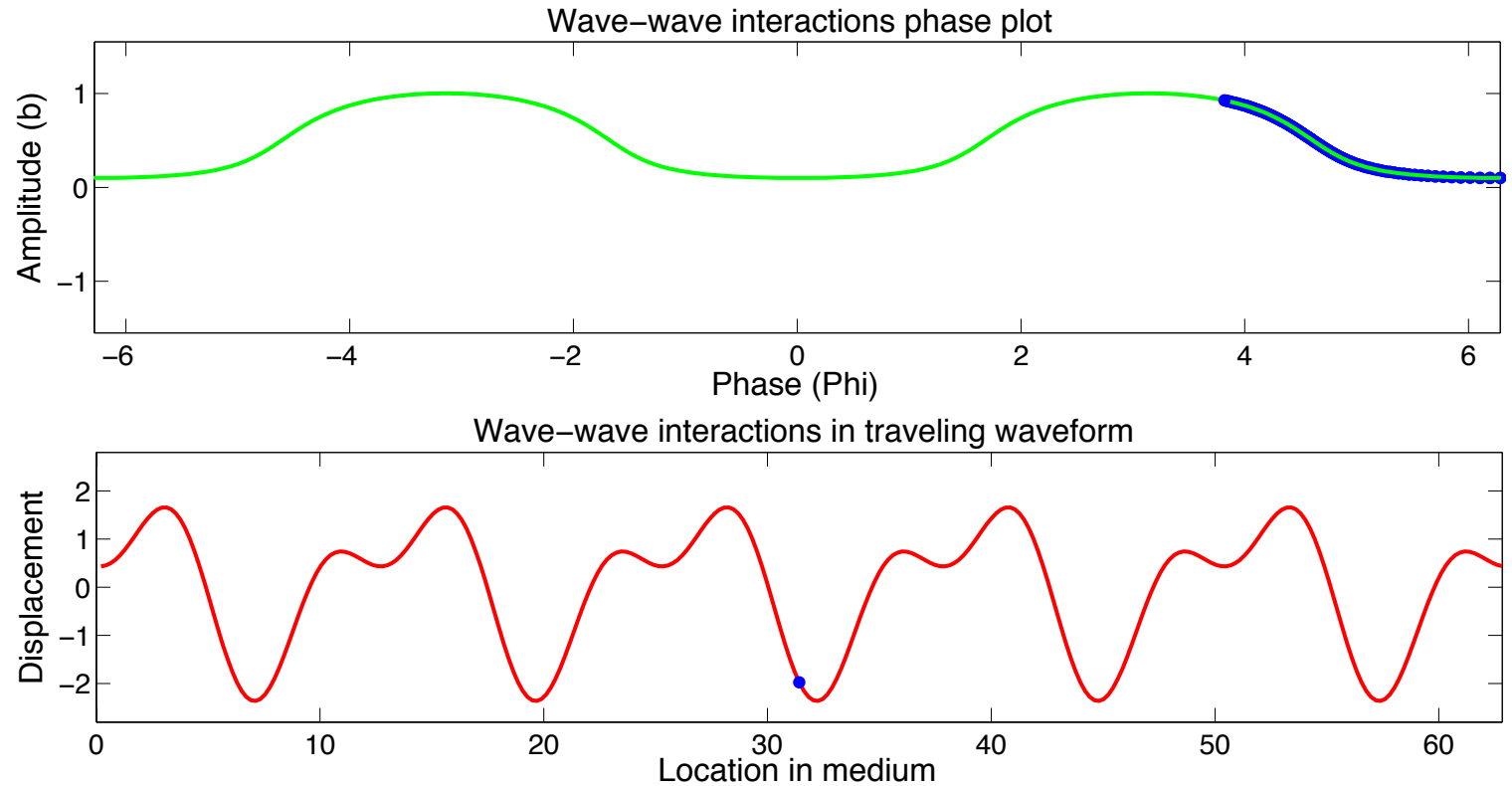

Fig. 9: This plot shows the phase plot corresponding to equation (24) and a snapshot of the time evolution of wave propagation in a chain for initial conditions $\phi_{0}=2 \pi$, and $b_{0}=0.1$. The green line represents a phase drift trajectory. The time evolution snapshot on the bottom plot corresponds to a point on the thick blue-green curve on the top figure. As this is a snapshot in time, the shape of the spatial wave would have changed in the next time step.

the center correspond to waves with a stronger shape variation, although still weakly locked. The complete simulation of one such case, at $b_{0}=-0.1$, is shown in Movie 3, which can be viewed from the attachments. Amplitudes and phases outside the separatrix lead to traveling waves with a shape-shifting behavior due to phase drift. A snapshot can be seen in Figure 9. The subsequent snapshots will reveal the shape-shifting behavior of the traveling waves as shown in the attached Movie 4.

TABLE I: Assumed Parameter Values for $\mu_{A_{0}}=0.5$

\begin{tabular}{lll}
\hline Parameter & Value & Description \\
\hline \hline$E$ & 2.0 & Total Characteristic Energy \\
$\sigma_{\omega}$ & 1.0 & Frequency Detuning \\
$\alpha$ & 1.0 & Linear Coefficient \\
$\beta$ & 1.0 & Quadratic Coefficient \\
$\epsilon$ & 0.1 & Nonlinearity Strength \\
\hline
\end{tabular}

TABLE II: Derived Parameter Values for $\mu_{A_{0}}=0.5$

\begin{tabular}{llll}
\hline Parameter & Value & Analytical Expression & Description \\
\hline \hline$\omega_{A_{0}}$ & 0.4948 & $\sqrt{2 \alpha\left(1-\cos \mu_{A_{0}}\right)}$ & Frequency of Wave-A \\
$\omega_{B_{0}}$ & 1.0896 & $2 \omega_{A_{0}}+\epsilon \sigma_{\omega}$ & Frequency of Wave-B \\
$\mu_{B_{0}}$ & 1.1523 & $\cos ^{-1}\left[1-\frac{\omega_{B_{0}}^{2}}{2 \alpha}\right]$ & Wave Number of Wave-B \\
$\sigma_{\mu}$ & 1.5232 & $\frac{1}{\epsilon}\left[\mu_{B_{0}}-2 \mu_{A_{0}}\right]$ & Wavenumber Detuning \\
$r$ & 0.5084 & $\frac{\omega_{A_{0}}\left[1-\cos \left(2 \mu_{A_{0}}\right)\right]}{2 \omega_{B_{0}}\left[1-\cos \left(\mu_{B_{0}}-\mu_{A_{0}}\right)\right]}$ & Elliptic Energy Coefficient \\
\hline
\end{tabular}

\section{B. Emergent Waveforms}

The analysis suggests the possibility of the existence of emergent wave harmonics. A real harmonic wave, and likewise a simulated nonlinear wave, will likely have impurities that can be expressed in terms of wave mode perturbations. Due to quadratic nonlinearity, a very small amplitude subharmonic or superharmonic wave mode can slowly drift in its phase according to the plot in Figure 2, until it reaches the separatrix near $\phi= \pm \pi$, at which point the waves have a burst of larger amplitude as it circumnavigates the separatrix. Depending on the parameters and wave numbers, the amplitude of this emergent wave burst can have varying significance. If the quadratic influence is weak, this emergent burst would be generated on a long time 


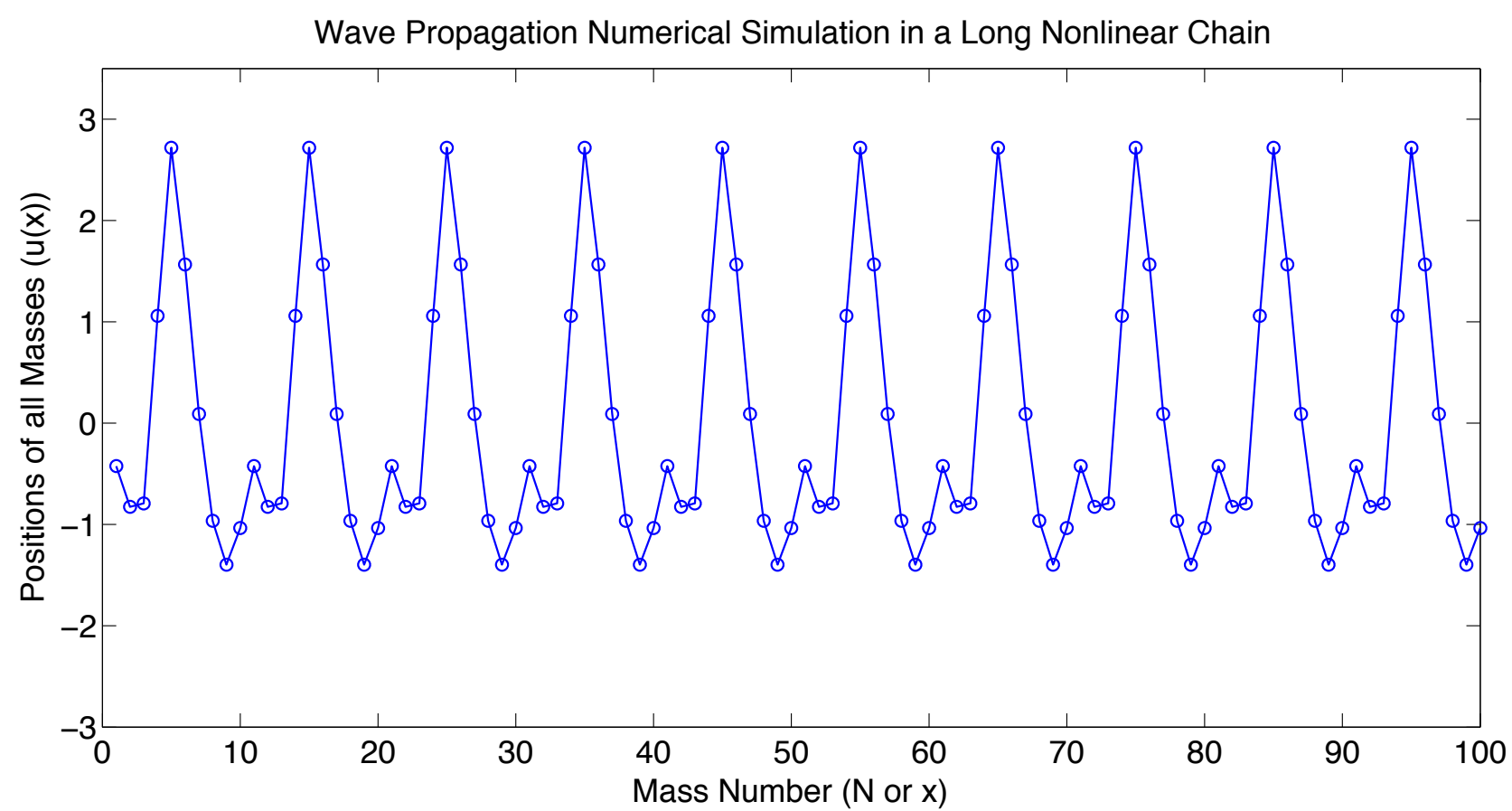

Fig. 10: A snapshot from the numerical simulation of the weakly phase-locked case of the mass displacement in a mass-spring chain with 100 masses with quadratic nonlinearity for $\mu_{A_{0}}=\frac{\pi}{5}$ and the initial conditions $b_{0}=0.85$ and $\phi_{0}=0$.

scale. The wave-wave interaction occurs between 2:1 waves with a detuning parameter. Hence, a narrowband spectrum of emergent wave bursts could be possible.

TABLE III: Initial Conditions of State-Space Simulations for $\mu_{A_{0}}=0.5$

\begin{tabular}{lll}
\hline Figure No. & Waveform & Initial Conditions for the Example Figures \\
\hline \hline Figure 5 & Phase-Locked & $\phi_{0}=0, \theta_{A_{0}}=0, \theta_{B_{0}}=0, b_{0}=-0.5, a_{0}=1.8553$ \\
Figure 6 & Phase-Locked & $\phi_{0}=0, \theta_{A_{0}}=0, \theta_{B_{0}}=0, b_{0}=1.3, a_{0}=0.7809$ \\
Figure 7 & Weakly Phase-Locked & $\phi_{0}=0, \theta_{A_{0}}=0, \theta_{B_{0}}=0, b_{0}=-0.3, a_{0}=1.9383$ \\
Figure 8 & Weakly Phase-Locked & $\phi_{0}=0, \theta_{A_{0}}=0, \theta_{B_{0}}=0, b_{0}=1.4, a_{0}=0.2805$ \\
Figure 9 & Phase-Drift & $\phi_{0}=2 \pi, \theta_{A_{0}}=0, \theta_{B_{0}}=-2 \pi, b_{0}=0.1, a_{0}=1.9784$ \\
\hline
\end{tabular}

TABLE IV: Derived Parameter Values in the Chain Simulation for $\mu_{A_{0}}=\pi / 5$

\begin{tabular}{llll}
\hline Parameter & Value & Analytical Expression & Description \\
\hline \hline$\omega_{A_{0}}$ & 0.6180 & $\sqrt{2 \alpha\left(1-\cos \mu_{A_{0}}\right)}$ & Frequency of Wave-A \\
$\omega_{B_{0}}$ & 1.4631 & $2 \omega_{A_{0}}+\epsilon \sigma_{\omega}$ & Frequency of Wave-B \\
$\mu_{B_{0}}$ & 1.1523 & $\cos ^{-1}\left[1-\frac{\omega_{B_{0}}^{2}}{2 \alpha}\right]$ & Wave Number of Wave-B \\
$\sigma_{\mu}$ & 2.0649 & $\frac{1}{\epsilon}\left[\mu_{B_{0}}-2 \mu_{A_{0}}\right]$ & Wavenumber Detuning \\
$r$ & 0.4862 & $\frac{\omega_{A_{0}}\left[1-\cos \left(2 \mu_{A_{0}}\right)\right]}{2 \omega_{B_{0}}\left[1-\cos \left(\mu_{B_{0}}-\mu_{A_{0}}\right)\right]}$ & Elliptic Energy Coefficivent \\
\hline
\end{tabular}

The $(b, \phi)$ phase space dynamics in Figure 2 is an asymptotic approximation of the strain dynamics in the nonlinear chain. The resulting slow flow is represented by center (weakly locked), saddles, and whirls (drift). The saddles are connected by heteroclitic orbits. This is a structurally unstable flow. Structural instability means that an arbitrarily small perturbation will cause a qualitative change in phase space. The system has been reduced to a smooth 2-D flow, in terms of $b$ - $\phi$, which contains non-hyperbolic fixed points (centers) and saddle connections (heteroclitic orbits). Both of these features indicate structural instability via Peixoto's theorem [21].

The original system is a small perturbation of the $(a, b, \phi)$ system; and that small perturbation can disrupt the structurally unstable representation of $(a, b, \phi)$. It is conceivable that the full system may exhibit homoclinic tangles and all of its implications such as chaos, fractals, and strange attractors. 

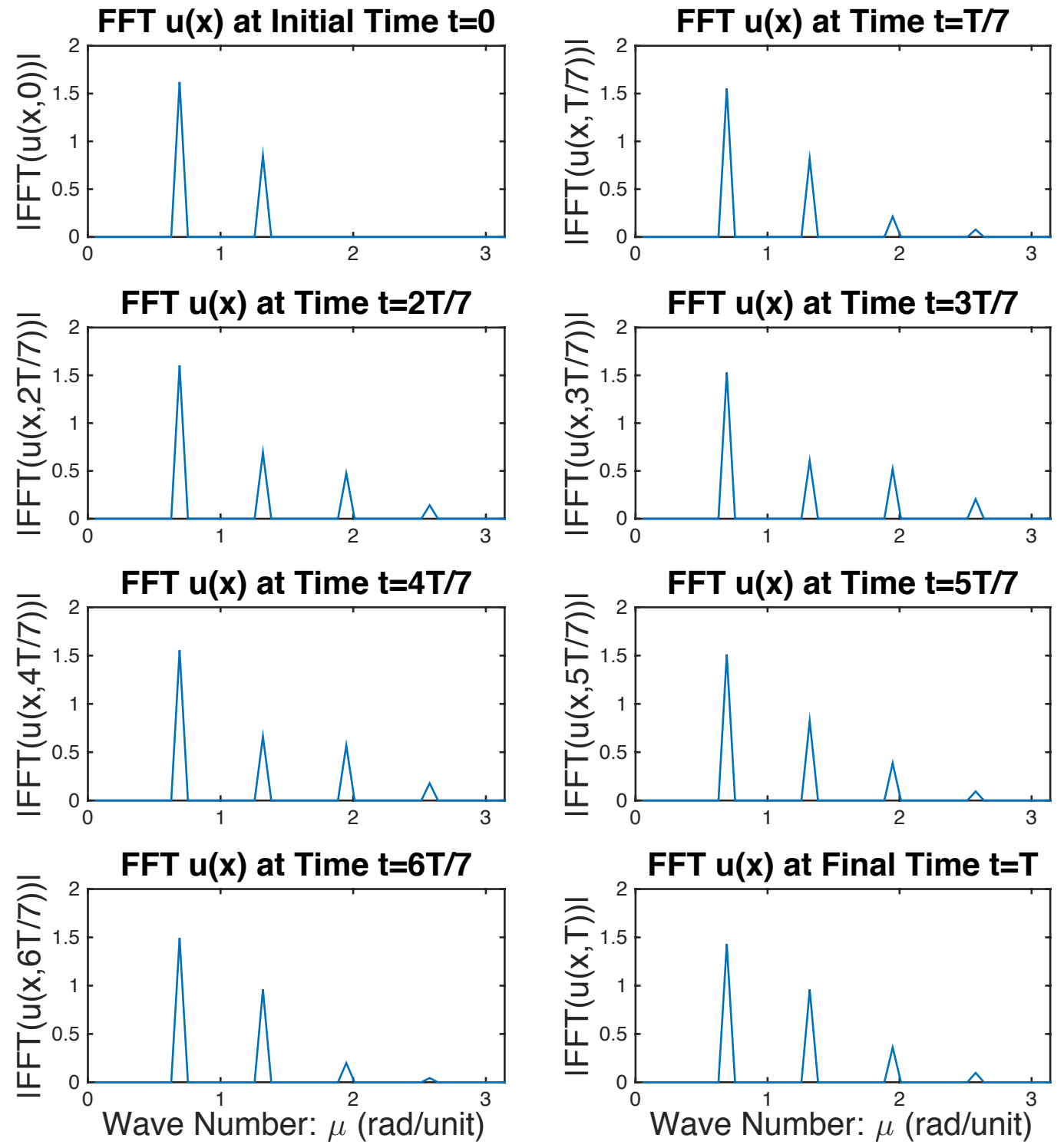

Fig. 11: Spatial FFTs of a numerical simulation, representing a weakly phase locked case, of a mass-spring chain with 100 masses with quadratic nonlinearity for $\mu_{A_{0}}=\frac{\pi}{5}, T=23 \mathrm{sec}$ and the initial conditions $b_{0}=0.85$ and $\phi_{0}=0$.

In case of three-wave interactions when the frequencies and the wave numbers are in a 1:2:4 ratio, it can be derived that the amplitudes of these three waves lie on a three-dimensional ellipsoid corresponding to a particular energy state.

The equations (21) to (24) and the solutions in equation (28), show that the strength of the quadratic nonlinearity affects the rate at which the energy is exchanged. For different energy states the amplitudes of these waves are defined in different concentric ellipses for given detuning parameter. Given the detuning parameter, the frequency of one of the waves, and the strength of the nonlinearity, one can find the corresponding frequency and wave numbers of the other sub- or the super-harmonic wave.

\section{Numerical Simulations of the Full Chain}

In this section we present numerical simulations performed directly on a quadratic mass chain to demonstrate some of the behavior predicted by the analysis in the previous section on the slow-flow dynamics derived by multiple scales. In place of an infinite chain, we simulate the equations of motion (equation (5) with $\alpha=1.0, \beta=1.0$ and $\gamma=0$ ) for a chain of 50 or 100 masses connected such that the chain is circular, i.e. the last mass is connected with the first mass. The wavenumber was chosen such that the fundamental wavelength was 10 units per cycle, such that an integer number of waves covered the entire chain. The simulations were done with the parameters in Table I, except that the wave number is $\mu_{A_{0}}=\pi / 5$, corresponding to the 

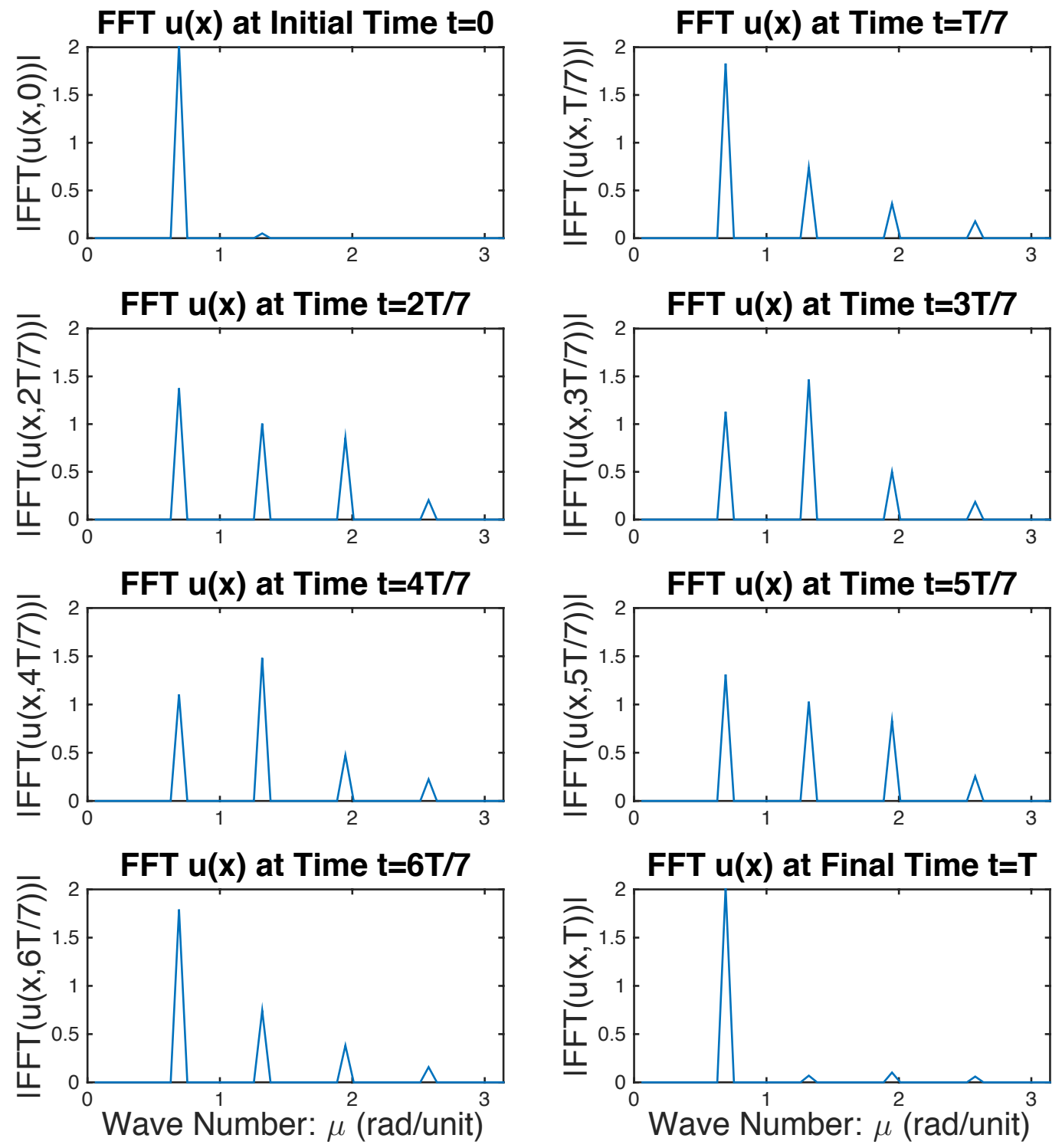

Fig. 12: Spatial FFTs of a numerical simulation, representing a phase drift (or very weakly locked) case, of a mass-spring chain with 100 masses with quadratic nonlinearity for $\mu_{A_{0}}=\frac{\pi}{5}, T=56 \mathrm{sec}$ and the initial conditions $b_{0}=0.05$ and $\phi_{0}=0$.

given wavelength. This results in derived parameter values shown in Tabletable:DerivedParametersNumSim. The corresponding $(b, \phi)$ phase plot with these parameters is qualitatively similar to that of Figure 2. The free wave is initiated by setting initial conditions. The displacement initial conditions for the wave simulation were chosen as $z_{0}=a \cos \left(\mu_{A_{0}} j\right)+b \cos \left(2 \mu_{A_{0}} j\right)$, and the velocity initial conditions as $v_{0}=a \omega_{A_{0}} \sin \left(\mu_{A_{0}} j\right)+2 b \omega_{A_{0}} \sin \left(2 \mu_{A_{0}} j\right)$.

Figure 10 shows a snapshot of a weakly locked waveform, which can be viewed in Movie 5. As this is a snapshot in time, at another snapshot, the shape of the spatial wave will have shifted. The movie shows the shape shifting of the traveling wave. In this case, the amplitude, $b$, of the secondary wave follows a small periodic fluctuation, such as in the closed curves surrounding a fixed point of center type in Figure 2. The amplitude $a$ of the primary wave thus fluctuates according to equation (23). Figure 11 shows the spatial fast Fourier transforms (FFTs) of snapshots of the chain at equal intervals in time through the simulation period $T$, which covers one cycle of the periodic orbit in the corresponding $(b, \phi)$ phase space, including the beginning time, $t=0$, and end time, $t=T$. Looking at this figure, it can be seen that the secondary amplitude varies from $b=0.85$, decreases somewhat, and then increases nearly to its initial value. Meanwhile, $a$ fluctuates slightly to "preserve" total energy. Some energy, however, gets channeled into the emergence of harmonics at $3 \mu_{A_{0}}$ and $4 \mu_{A_{0}}$. This occurs for two reasons. One is that the perturbation analysis is an asymptotic approximation with two single-harmonic parts, and the initial conditions of the waveform thus include only these two harmonics. But the ensuing free response is nonlinear, and involves 

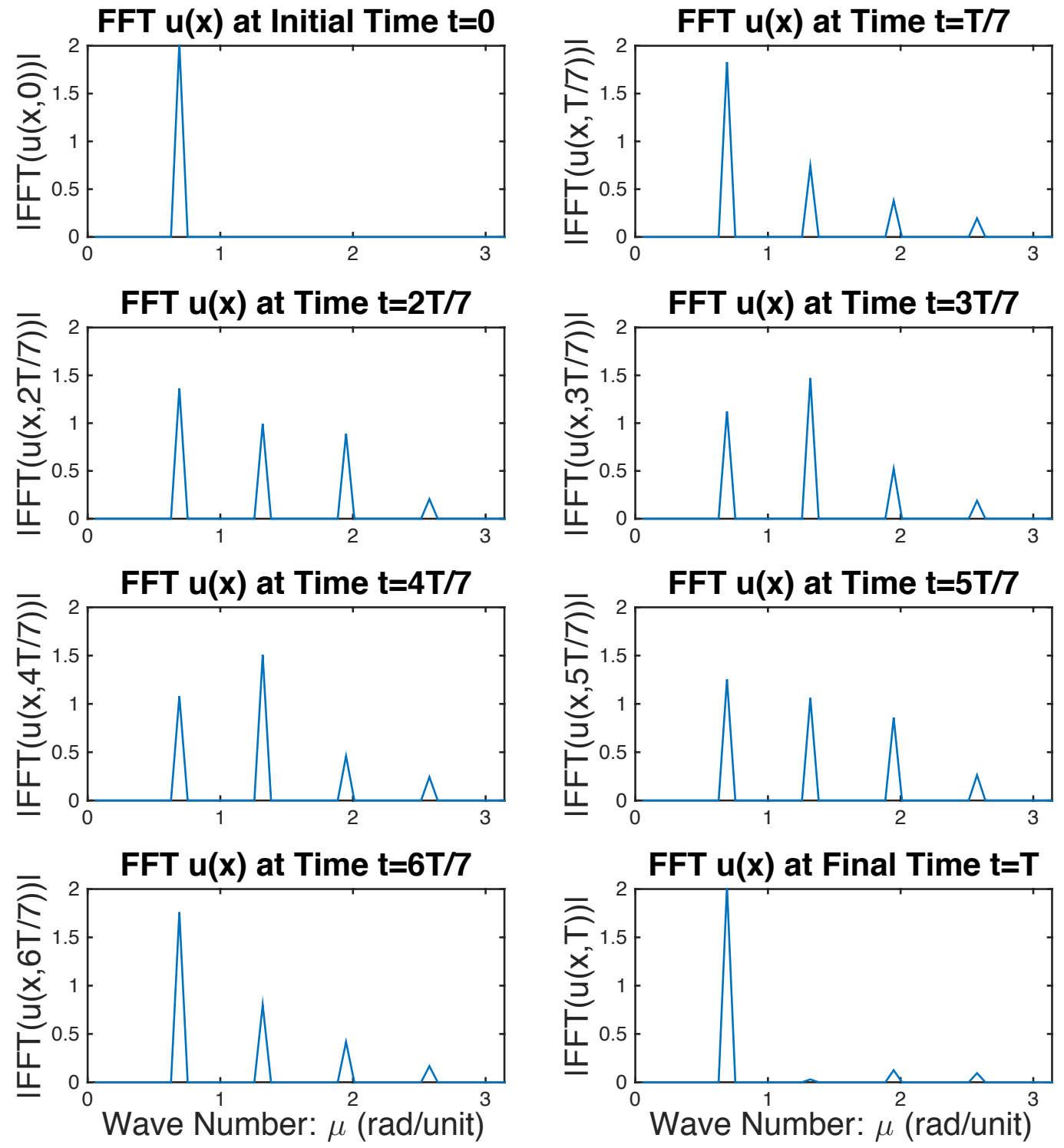

Fig. 13: Spatial FFTs of a numerical simulation, representing an emergent case, of a mass-spring chain with 100 masses with quadratic nonlinearity for $\mu_{A_{0}}=\frac{\pi}{5}, T=57 \mathrm{sec}$ and the initial conditions $b_{0}=0$ and $\phi_{0}=0$.

higher harmonics, which thus develop after the initial conditions are set. Furthermore, there are wave-wave interactions between these higher-order harmonics and the primary components. In this example, we can see the interaction between the B-wave and its second harmonic (wave number at about 2.6), which starts at zero and returns nearly to zero when $t=T$. The emergent wave at $3 \mu_{A_{0}}$ is a nonlinear effect, but is not involved in this leading order analysis of the quadratic wave-wave interactions. (A quadratic wave-wave interaction with this harmonic of order 3 that would be described by this first-order perturbation analysis would involve an order at 1.5 or 6 , both of which are not represented in this waveform. A higher-order perturbation analysis might reveal a relevant interaction.)

Figure 12 shows the spatial FFT progression for the case of a waveform in phase drift (or possibly very weakly locked), shown in Movie 6. The corresponding state space feature in Figure 2 is that of an orbit for which $b$ is very small. Based on equation (24), $\phi^{\prime}$ will be large when $b$ is small, and so the trajectory is fast and quickly reaches the saddle and then circumnavigates the separatrix, at which time the value of $b$ is larger, $\phi^{\prime}$ is smaller, and the trajectory is slow. (The behavior is very similar whether the trajectory circumnavigates very near the separatrix or the inside or the outside.) Thus, $b$ spends a longer part of the cycle being large than small. The speed of the trajectories are indicated by the small arrows in Figure 2 . These trends are seen in the FFT progression. The initial amplitudes are $b_{0}=0.05$ and $a_{0} \approx 2$. The amplitude, $b$, of Wave-B quickly reaches values in the range of 0.8 to 1.5 , holds these values for most of the cycle, and returns to a value of $b=0.05$ 
by the end of the cycle. The FFT progression again shows additional emergent higher harmonics.

The last case, shown in Figure 13, demonstrates an emergent waveform (Movie 7). In this case, the initial wave amplitudes are $b_{0}=0$ and hence $a_{0}=2$ based on equation (23). Wave-B quickly emerges (as do higher harmonics) because of the higherorder nonlinear effects, persists for much of the cycle, becoming the dominant wave, and returns to an amplitude of nearly zero. Then the cycle repeats itself. This waveform follows a phase drift or very weakly locked pattern depending $\mathrm{n}$ whether the wave energies inside or outside the separatrix, similar to the previous example, except that the emergent superharmonic waveform is generated from a single wave.

We did not observe an emergent subharmonic waveform. Based on state-space dynamics of the slow flow, if we have $b_{0} \neq 0$ and $a_{0}=0$, there can be a fixed point according to equation (29) of saddle type. If $a_{0}=0, b_{0}$ has its maximum value. This initial condition is on the stable manifold of the fixed point. The trajectory will approach the fixed point unless noise or nonlinear perturbation bumps it off of the stable manifold.

\section{CONCLUSION}

In this paper, a detailed study of interactions of two traveling waves in an infinite nonlinear uniform chain with weak quadratic nonlinear elements has been presented. With first-order multiple-scales analysis, solutions for slowly varying amplitude and phase relations as well as the fixed points and the stability of the wave propagation were established. This analysis, along with numerical simulations of the full mass-spring chain, uncovered an energy exchange between the two propagating waves through the chain with drifting phase or weakly locking phase. This energy exchange has a similar mechanism as that in an elastic pendulum.

The analysis shows that when two waves in a 2:1 resonance condition are propagating in a uniform infinite chain with weak quadratic nonlinear elements, they create phase drift or weak phase locking while an energy exchange between the two waves occurs on an ellipse in amplitude space. At a fixed point in the amplitudes and phase, the traveling waveform has a fixed shape without any phase drift. The dynamics of the amplitude and phase consists of oscillations and whirls separated by a separatrix. The phase drifts are seen outside the separatrix. Weak phase locking and phase drift both produce shape-shifting traveling waves. The shape shifting can be more extreme in the phase drift case. The observed behavior depends on initial conditions and parameters. The state-space dynamics of the wave amplitude and phase suggests the possibility of emergent waves. The emergent harmonics are seeded by noise or nonlinear effects, and are injected into the emergent mechanism, which has phase drift. These behaviors were validated through the numerical simulations.

We conjecture that emergent harmonics can occur in any system with sustained waves with quadratic nonlinearity. The strength of the quadratic nonlinearity affects the time scale of the energy exchange, and hence the emergence. Very weak quadratic effects may generate energy exchanges on time scales slower than that of wave dissipation, depending on the damping and nonlinearity in the system.

A second-order perturbation analysis, in which the slow flow is expected to be difficult to solve analytically, could reveal the effects of both quadratic and cubic nonlinearities.

\section{ACKNOWLEDGMENTS}

This material is based upon work supported by the National Science Foundation under Grant No. CMMI-1030377. Any opinions, findings, and conclusions or recommendations expressed in this material are those of the authors and do not necessarily reflect the views of the National Science Foundation.

\section{REFERENCES}

[1] Asfar, O. R., and Nayfeh, A. H., 1983. "The application of the method of multiple scales to wave propagation in periodic structures". Society of Industrial and Mathematics Review, 25(4), pp. 455-480.

[2] Chakraborty, G., and Mallik, A. K., 2001. "Dynamics of a weakly nonlinear periodic chain". International Journal of Non-linear Mechanics, 36(2), pp. 375-389.

[3] Rushchitsky, J. J., and Savel'eva, E. V., 2006. "On the interaction of cubically nonlinear transverse plane waves in an elastic material". International Applied Mechanics, 42(6), pp. 661-668.

[4] Jang, J. H., Ullal, C. K. U., Gorishnyy, T., Tsukruk, V. V., and Thomas, E. L., 2006. "Mechanically tunable three-dimensional elastomeric network/air structures via interference lithography". Nano Letters, 6(4), pp. 740-743.

[5] Marathe, A., and Chatterjee, A., 2005. "Wave attenuation in nonlinear periodic structures using harmonic balance and multiple scales.". Journal of Sound and Vibration, 289(4-5), pp. 871-888.

[6] Narisetti, R. K., Leamy, M. J., and Ruzzene, M., 2010. "A perturbation approach for predicting wave propagation in one-dimensional nonlinear periodic structures". ASME Journal of Vibration and Acoustics, 132(3), p. 031001.

[7] Narisetti, R. K., Ruzzene, M., and Leamy, M. J., 2011. "A perturbation approach for analyzing dispersion and group velocities in two-dimensional nonlinear periodic lattices". ASME Journal of Vibration and Acoustic, 133(6), p. 061020.

[8] Rothos, V. M., and Vakakis, A. F., 2009. "Dynamic interactions of traveling waves propagating in a linear chain with an local essentially nonlinear attachment". Wave Motion, 46(3), pp. 174-188.

[9] I., H. M., Hulbert, G., and Scott, R., 2002. "Tailoring of wave propagation characteristics in periodic structures with multilayer unit cells". Proceedings of 17th American Society of Composites Technical Conference.

[10] I., H. M., Hamza, K., Hulbert, G., and Saitou, K., 2007. "Optimal synthesis of 2d phononic crystals for broadband frequency isolation". Waves Random Complex Media, 17(4). 
[11] Sigmund, O., and Jensen, J. S., 2003. "Systematic design of phononic band-gap materials and structures by topology optimization". Philosphical Transactions of the Royal Society A: Mathematical, Physical and Engineering Sciences, 361(1806), pp. 1001-1019.

[12] Diaz, A., Haddow, A., and Ma, L., 2005. "Design of band-gap grid structures". Structural and Multidisciplinary Optimization, $29(6)$, pp. 418-431.

[13] Rushchitsky, J. J., 1999. "Interaction of waves in solid mixtures". Applied Mechanics Reviews, 52(2), pp. 35-74.

[14] Rushchitsky, J. J., and Chattani, C., 2004. "Evolutionequationsforplane cubically nonlinear elastic waves". International Applied Mechanics, 40(1), pp. 70-76.

[15] Kittel, C., 1976. Introduction to Solid State Physics. 5th edition, Wiley, New York.

[16] Kaajakari, V., Mattila, T., Oja, A., and SeppŁ, H., 2004. "Nonlinear limits for single-crystal silicon microresonators". IEEE Journal of Micro-ElectroMechanical Systems, 13(5), pp. 715-724.

[17] Kaajakari, V., Mattila, T., Lipsanen, A., and Oja, A., 2005. "Nonlinear mechanical effects in silicon longitudinal mode beam resonators". Sesors and Actuators A: Physical, 120(1), pp. 64-70.

[18] Manktelow, K., Leamy, M. J., and Ruzzene, M., 2011. "Multiple scales analysis of wave-wave interactions in a cubically nonlinear monoatomic chain". Nonlinear Dynamics, 63, pp. 193-203.

[19] Panigrahi, S. R., Feeny, B. F., and Diaz, A. R., 2013. "Second-order perturbation analysis of low amplitude traveling waves in a periodic nonlinear chain". Proceedings of the ASME International Design Engineering Technical Conferences, 25th Conference on Mechanical Vibration and Noise, Portland, OR, August 4-7, ASME Paper No. DETC2013/VIB-13207.

[20] Nayfeh, A. H., 2011. "Introduction to perturbation techniques". John Wiley \& Sons.

[21] Guckenheimer, J., and Holmes, P., 1983. Nonlinear Oscillations, Dynamical Systems, and Bifurcations of Vector Fields. Springer-Verlag, New York. 


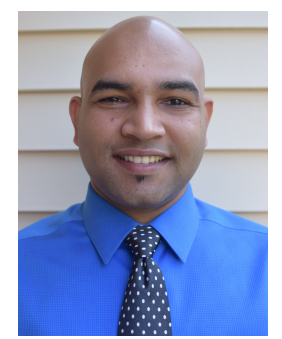

Dr. Smruti R. Panigrahi is currently working as a Scientist/Research Engineer at Ford Motor Company. He earned his M.S. in Applied Mathematics and Ph.D. in Mechanical Engineering from Michigan State University (MSU) in 2013 and 2014 respectively, He also holds a masters' degree in Mechanical Engineering from Washington State University and B.S. in Manufacturing Science and Engineering from the Indian Institute of Technology, Kharagpur, India. Dr. Panigrahi's research interests are dynamics and vibration, renewable energy, metamaterials design, deep/machine learning, (big)data science, vehicle dynamics and control, computational intelligence, smart-mobility, and intelligent transportation systems.

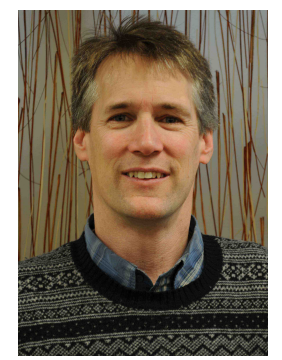

Prof. Brian F. Feeny earned his Ph.D. in Nonlinear Dynamics and Chaos from Cornell University in 1990. He then won a fellowship to engage in postdoctoral research at ETH Zurich, Switzerland. He has been serving as a professor at Michigan State University since 1992. He is a member and fellow of American Society of Mechanical Engineers (ASME). He has also served as the Chair of ASME Technical Committee on Vibration and Sound and as associate editor of ASME Journal of Vibration and Acoustics. His research interests are in nonlinear dynamics and vibration, chaos, fractals, wave propagation, system identification, proper orthogonal decomposition, and energy scavenging.

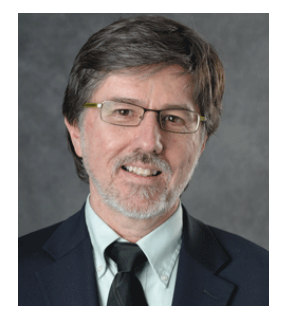

Prof. Alejandro R. Diaz earned his B.S.E., M.S., and Ph.D. in Aerospace Engineering from University of Michigan in 1978, 1979, and 1982 respectively. Since then he has been serving as a professor at Michigan State University. He is a member and fellow of American Society of Mechanical Engineers. He also served as the head of the Mechanical Engineering Department at MSU from 2010 to 2015. His research interests are in topology optimization, optimal design of structures and materials, design of bandgap structures, origami inspired frequency selective surfaces, finite element methods, metamaterials, and energy scavenging. 